\title{
Was ist Intuition? Grundlagen zu einem umfassenden Intuitionsverständnis
}

Ziel des folgenden Kapitels ist es, ein Verständnis der Intuition als Erkenntnisgattung zu gewinnen. Dafür gilt es zunächst die Gemeinsamkeiten verschiedener bestehender Intuitionsverständnisse zu erkennen. Ein wiederkehrendes Motiv sind hierbei die Eigenschaften der Unmittelbarkeit und Gewissheit. Diese erscheinen sowohl in den im vorigen Kapitel dargestellten Intuitionsverständnissen der neuzeitlichen Philosophie als auch in den heute und insbesondere durch die psychologische Forschung geläufigen Intuitionsverständnissen. Hier muss zunächst geklärt werden, welche Art von Eigenschaften >Unmittelbarkeit « und `Gewissheit< sind. Als plausibel wird sich dabei erweisen, diese als phänomenale Eigenschaften mentaler Zustände zu bezeichnen. Diese phänomenalen Eigenschaften stellen die entscheidende Verbindung zwischen mentalen Zuständen her, welche sie als der Erkenntnisgattung der Intuition zugehörig gelten lassen. Im Weiteren soll dafür argumentiert werden, dass die Intuition Neigungen zu Überzeugungen konstituiert, ohne dabei selber auf den Bereich des Propositionalen reduziert werden zu können. Intuition spielt sich im Wesentlichen auf der nichtpropositionalen Ebene ab; Intuitionen haben daher primär nichtbegrifflichen Erfahrungsgehalt. Diese These wird neben der phänomenalen auch durch kognitionswissenschaftliche Evidenz, namentlich durch Verweise auf die Dual-Prozess-Theorie sowie auf die Theorie der mentalen Modelle, gestützt. Das Verhältnis zwischen der nichtpropositionalen und der propositionalen Ebene im Bereich der Intuition wird auszuloten sein.

Das durch die genannten Ausführungen zu erzielende Intuitionsverständnis kann als Orientierungsrahmen für die später folgende Untersuchung der intuitiven Rechtfertigung dienen. Ebenso wird dieses Intuitionsverständnis verschiedene wichtige Abgrenzungen erlauben: zunächst vom Intuitions-Apriorismus, sowie von Theorien, die Intuitionen als Erscheinungen suigeneris betrachten. Außerdem kann eine Reduktion der Intuition auf Wahrnehmung oder auf Zustände ohne repräsentationalen Gehalt zurückgewiesen werden. Für alle diese Schritte dient das hier vorgelegte Intuitionsverständnis als unentbehrliche Grundlage.

Das soeben skizzierte Vorgehen setzt eine methodische Annahme in Bezug auf die Aufgabe der Begriffsbestimmung voraus, die zunächst explizit gemacht 
werden soll. Angesichts der vielfältigen Verwendungsweisen des Intuitionsbegriffs, die zudem einem starken historischen Wandel unterworfen sind, ist es aussichtslos nach einem Merkmal zu suchen, welches allen Intuitionen gemeinsam ist. Daher soll im Folgenden von Wittgensteins Konzept der >Familienähnlichkeit< ausgegangen werden. ${ }^{92}$ Den folgenden Ausführungen liegt somit die Annahme zugrunde, dass sich die verschiedenen mit >Intuition< bezeichneten Phänomene zwar in vielen Belangen ähneln, jedoch keine gemeinsame Schnittmenge dieser Ähnlichkeiten auszumachen ist. Wie können wir uns in solchen Fällen dem Verständnis eines Begriffs wie >Intuition< annähern? Hier ist ein Vorgehen zu wählen, wie es Wittgenstein in Bezug auf den Spielbegriff darstellt:

\begin{abstract}
Aber ist es sinnlos zu sagen: »Halte dich ungefähr hier auf!«? Denk dir, ich stünde mit einem Andern auf einem Platz und sagte dies. Dabei werde ich nicht einmal irgend eine Grenze ziehen, sondern etwa mit der Hand eine zeigende Bewegung machen - als zeigte ich ihm einen bestimmten Punkt. Und gerade so erklärt man etwa, was ein Spiel ist. Man gibt Beispiele und will, dass sie in einem gewissen Sinne verstanden werden. ${ }^{93}$
\end{abstract}

Ein solches Vorgehen bringt keine strenge Definition eines Begriffs, etwa im Sinne der Auflistung hinreichenderund notwendiger Bedingungen, hervor. Dies ist aber für die Zwecke dieser Arbeit gar nicht nötig. Statt einen Begriff exakt zu definieren geht es vielmehr darum, eine Klasse von mentalen Zuständen zu verstehen, die aufgrund ihrer Ähnlichkeit verbreitet mit >Intuition < bezeichnet werden. Diese einzugrenzende Klasse macht nur einen (wenn auch zentralen) Teil der Verwendungsweisen von >Intuition< aus; andererseits wird dieser Begriff wohl nicht konstant auf mentale Zustände der zu bestimmenden Klasse angewandt. Solange durch die Beispiele und Beschreibungen nachvollziehbar wird, um welche Klasse es sich handelt, stellen diese Einschränkungen kein Problem dar. Die Beispiele und Beschreibungen exemplifizieren zueinander ähnliche erkenntnisbezogene Zustände, bzw. vereinfachend: eine Erkenntnisgattung, auf welche die Betrachtung gerichtet werden soll.

\title{
3.1 Phänomenale Ebene: Unmittelbarkeit und Gewissheit
}

Bei allen Unterschieden weisen die im vorigen Kapitel vorgestellten philosophischen Positionen zur Intuition eine Gemeinsamkeit auf: Sie alle haben

92 Vgl. Wittgenstein, PU §65f.

93 Wittgenstein, PU $§ 71$. 
(wenn auch nicht immer in diesen Worten ausgedrückt) ein Intuitionsverständnis, welches den Intuitionen die Eigenschaften der Unmittelbarkeit und der Gewissheit zuordnet. Eine nähere Beschreibung dieser Eigenschaften kann direkt in Bezug auf Descartes' Intuitionsverständnis gegeben werden. Jedoch muss gegenüber dem Cartesischen Verständnis eine für die weitere Untersuchung wichtige Modifikation vorgenommen werden: Unmittelbarkeit und Gewissheit dürfen nicht mehr als Merkmale verstanden werden, die objektiv etwas über den epistemischen Status der Intuition aussagen, sondern müssen als phänomenale Eigenschaften der Intuition an das subjektive Erleben eines mentalen Zustands gebunden werden. Im Folgenden soll zunächst das klassisch-philosophische Verständnis - exemplarisch anhand Descartes - von Unmittelbarkeit und Gewissheit repetiert werden. Anschließend gilt es zu zeigen, inwiefern diese Eigenschaften auch für ein heute verbreitetes, psychologisch geprägtes Intuitionsverständnis zutreffend sind. Dabei wird die Notwendigkeit der erwähnten Modifikation deutlich. Diese soll dann explizit für Unmittelbarkeit und Gewissheit vorgenommen werden. Dabei muss noch präzisiert werden, was es heißt, einem mentalen Zustand phänomenale Eigenschaften zuzuschreiben.

Unter >Unmittelbarkeit< versteht Descartes, dass ein Erkenntnissubjekt Intuitionen nicht aus Gründen herleitet, sondern sich die Intuitionen schlagartig präsentieren. Beispielsweise folgt für Descartes >ich denke < im genannten Sinn unmittelbar aus >ich existiere<, was bedeutet, dass Descartes keine weiteren Gründe dafür angeben kann und muss, warum dies folgt. Es gibt keine deduktives Argument, welches uns hier von der ersten zu der zweiten Aussage bringt. ${ }^{94}$ Die Intuition als unmittelbar und somit Tatsachen schlagartig erfassend zu verstehen, hat eine Tradition die bis zu Epikurs epibolé zurückreicht. Diese Tradition dürfte entscheidend dafür gewesen sein, dass epibolé in Latein zu intuitio wurde und bis heute Analogien zwischen Intuition und Wahrnehmung gebildet werden. Das schlagartige oder auch ganzheitliche Erfassen, also quasi auf einen Blick (uno intuitu, wie es Spinoza beim Kaufmann-Beispiel nennt), stellt sowohl die Wahrnehmung als auch die Intuition in einen Gegensatz zu deduktiv hergeleiteten Überzeugungen.

Auch in heute verbreiteten und somit psychologisch geprägten Intuitionsverständnissen ist Unmittelbarkeit ein entscheidendes Merkmal. Dabei scheint Folgendes unter $>$ Unmittelbarkeit $<$ verstanden zu werden: Dem Subjekt sind die Gründe für das Erkannte nicht zugänglich, möge eine Ableitung aus Gründen unmöglich, möglich oder notwendig sein. Dies ist ein offeneres Verständnis von >Umittelbarkeitくals das Cartesische. Zwar lässt auch Descartes zu, dass 
manche Erkenntnisse sowohl auf intuitivem als auch auf deduktivem Weg zustande kommen können. Der intuitive Weg hat aber auch in diesen Fällen immer objektive Geltung. Am bekannten Beispiel wird dies klar: »Ich denke, ich existiere« ist eine gültige intuitive Erkenntnis, die auch objektiv keiner deduktiven Absicherung bedarf. Anders verhält sich dies in Bezug auf ein Verständnis von Unmittelbarkeit, welches an zeitgenössische psychologische und alltägliche Verwendungsweisen des Intuitionsbegriffs anschlussfähig ist. Die Geltung der intuitiven Erkenntnis ist hier für Aussenstehende nicht unbedingt nachvollziehbar. Die Gründe müssten möglicherweise deduktiv expliziert werden, damit der Schluss als gültig akzeptiert würde. Golfspieler, die intuitiv den richtigen Schläger wählen und Feuerwehrfrauen, die intuitiv gerade noch rechtzeitig ein brennendes Gebäude verlassen, sind gute Beispiele für a posteriori-Intuition, für welche Unmittelbarkeit im hier gemeinten subjektiven Sinn kennzeichnend ist. ${ }^{95}$ Die Golfspieler und Feuerwehrfrauen handeln aus Gründen, die ihrer Wahrnehmung der Situation entspringen, ihnen aber nicht bewusst zugänglich sind. Das Cartesische Verständnis von Unmittelbarkeit liess dagegen als Erkenntnisgegenstand der Intuition nur selbstevidente Wahrheiten zu, da nur diese unmittelbar im objektiven Sinn sind, also nie der deduktiven Absicherung bedürfen. Um den Anschluss an die psychologisch-alltäglichen Verständnisse zu erzielen, soll die Eigenschaft der Unmittelbarkeit beibehalten werden, jedoch nur unter einer expliziten Verschiebung des Verständnisses: >Unmittelbarkeit` soll nun bezeichnen, wie es für ein Subjekt ist, eine Intuition zu haben, beschreibt also eine phänomenale Eigenschaft des mentalen Zustandes Intuition.

Die zweite viele Intuitionsverständnisse von Descartes bis heute umspannende Eigenschaft der Intuition ist die Gewissheit. Dass die Eigenschaft der Gewissheit nicht nur im Zentrum des Cartesischen Intuitionsverständnisse, sondern wahrscheinlich im Zentrum aller Intuitionsverständnisse, einschließlich der psychologischen, steht, kann wie folgt plausibilisiert werden: Man stelle sich vor, jemand müsste einem ahnungslosen Gegenüber erklären, was >Intuition< bedeutet. So unterschiedlich diese Beschreibungen sein mögen, kaum eine wird auf das Element der Gewissheit verzichten, etwa indem man Intuition erklärt als »etwas, von dem du eine starke Ahnung hast, dass es so ist - aber du weisst nicht, wie du darauf kommst.« Inwiefern die Gewissheit auch bei Descartes zentrales Merkmal der Intuition ist, wurde in den Textstellen der Regulae bereits deutlich. Doch Descartes anerkennt auch den Unterschied zwischen Gewissheit und Sicherheit, insofern er es in späteren Arbeiten für notwendig erachtet, die in den Regulae noch als infallibel verstandenen 
Intuitionen deduktiv abzusichern. Dies bedeutet, dass die hohe (subjektive) Gewissheit der Intuition noch keine (objektive) Sicherheit impliziert. Mit dieser Konzeption von Gewissheit, welche sie klar von Sicherheit abgrenzt, bleibt die Möglichkeit der Fallibilität der Intuition offen, was für ein aktuelles, an Psychologie und Umgangssprache anschlussfähiges Intuitionsverständnis unerlässlich ist.

Da jedoch >Gewissheit< oft einer objektiven Deutung unterliegt, ist es wichtig, auch hier die Modifikation explizit zu machen und so den bei Descartes eingeschlagenen Weg konsequent weiterzugehen: Gewissheit soll keinesfalls mehr als die Intuition objektiv auszeichnende Eigenschaft verstanden werden, sondern - wie auch die Unmittelbarkeit - als phänomenale Eigenschaft der Intuition als mentaler Zustand.${ }^{96}$ Unter >Gewissheit< soll daher verstanden werden, dass das intuitiv Erkannte für das Subjekt in hohem Maß epistemisch attraktiv ist, wobei Attraktion hier meint, dass das Subjekt ohne gegenläufige Gründe das intuitiv Erkannte mit keiner oder nur geringer Zurückhaltung für wahr halten wird. Zu präzisieren ist hierbei, dass unter einem gegenläufigen Grund nicht nur eine der Intuition widersprechende Überzeugung, sondern etwa auch ein mangelndes Vertrauen in die eigene Intuition verstanden werden kann.

Dadurch, dass mit dem modifizierten Verständnis von Unmittelbarkeit und Gewissheit die Fallibilität der Intuition eingeräumt wird, muss bereits an dieser Stelle die Spinozistisch-Goethesche Einordnung der Intuition als höchste Erkenntnisgattung, die einen privilegierten Zugang zur Wahrheit ermöglicht, aufgegeben werden. Dies passt dazu, dass wir heute den Menschen als hinsichtlich seiner Erkenntnismöglichkeiten prinzipiell begrenzt verstehen; hinter diese Kantische Einsicht, die durch die modernen wissenschaftlichen Erkenntnisse weiter gestützt wurde, soll nicht zurückgegangen werden. Einen Königsweg der sicheren Erkenntnis gibt es nicht. Es ist leicht nachvollziehbar, dass Unmittelbarkeit und Gewissheit als subjektive Eigenschaften der Intuition zu der Vorstellung Anlass gegeben haben, die Intuition sei auch objektiv betrachtet eine privilegierte Erkenntnisart. Gerade die Verbindung der beiden subjektiven Eigenschaften erzeugt eine starke Motivation für solche Erhöhungen: Wenn das Gefühl der Gewissheit mit Unmittelbarkeit, also der Nicht-Angebbarkeit von Gründen, verbunden auftaucht, liegt die

96 Cohen spricht von einem »Gefühl des Glaubens« (credal feeling) im Zusammenhang mit einer Überzeugung (vgl. Cohen 1992, S. 9ff.; siehe dazu auch Kriegel 2015, S. 32). Es scheint plausibel, dass dieses credal feeling auch das Merkmal der Intuition ist. Die Gewissheit als phänomenales Merkmal der Intuition kann genau dieses Gefühl bezeichnen, wobei die hinzutretende Unmittelbarkeit die Intuition von einer (deduktiv erlangten) Überzeugung unterscheidet. 
Vermutung nahe, unbezweifelbare Erkenntnisse gewonnen zu haben. Dies mag bei Intuitionen im Bereich des Apriorischen, etwa bei selbstevidenten Wahrheiten, auch tatsächlich der Fall sein. Die Intuition aber auf diesen Bereich beschränkt zu sehen, würde es verunmöglichen, zu einem adäquaten Intuitionsverständnis zu gelangen, welches verbreiteten Verwendungsweisen des Intuitionsbegriffs gerecht wird. Andererseits sollten wir die Kernmerkmale der Intuition nicht einfach zurückweisen, da sie bis heute für den Intuitionsbegriff entscheidend sind. Die explizite Einordnung von Unmittelbarkeit und Gewissheit als phänomenale Eigenschaften der Intuition erlaubt es, diese Kernmerkmale beizubehalten, ohne eine Verpflichtung auf Infallibilität einzugehen.

Mit der Bezugnahme auf phänomenale Eigenschaften ergibt sich ein Anschluss zu aktuellen Diskussionen der kognitiven Phänomenologie. Zwei Verbindungen zu dieser Diskussion sollen genannt werden, da dies Aufschluss darüber geben kann, inwiefern auch der Intuition als mentaler Zustand phänomenale Eigenschaften zugeschrieben werden können. Zugleich werden diese Verbindungen für die Frage der intuitiven Rechtfertigung wichtig sein.

Erstens ist darauf hinzuweisen, dass durchaus umstritten ist, ob alle mentalen Zustände oder deren Gehalte phänomenale Eigenschaften haben. Eine restriktive Position lässt phänomenale Eigenschaften nur für mentale Akte, bzw. noch restriktiver, nur für Sinneswahrnehmungen zu. Demnach lässt sich etwa sinnvoll fragen, wie es ist, eine Rotwahrnehmung zu haben, jedoch nicht, wie es ist, eine Überzeugung zu haben. Gegen diese restriktive Position wenden Vertreter der kognitiven Phänomenologie unter anderem mit dem Argument des phänomenalen Kontrastes ein, dass auch Überzeugungen Phänomenalität aufweisen. Ein solcher Kontrast lässt sich beispielsweise feststellen, wenn man sich vergegenwärtigt, wie es für eine französischsprachige Person ist, einen französischen Satz zu hören, im Unterschied zu der entsprechenden Erfahrung einer nicht-französischsprachigen Person. Nur bei der französischsprachigen Person stellt sich ein »Gefühl des Verstehens« ein, was auf eine phänomenale Eigenschaft des Verstehens als mentaler Akt hindeutet, die bei der anderen Person nicht präsent ist. ${ }^{97}$ Weitere phänomenale Kontraste lassen sich feststellen, wenn wir aus der Ersten-Person-Perspektive zwei unterschiedliche Einstellungen zu einem Gedanken miteinander vergleichen, so etwa das Urteil, dass $p$ mit dem bloßen Unterhalten eines Gedankens, dass $p .{ }^{98}$ In Bezug auf die Intuition ist es äußerst plausibel, ebenfalls solche phänomenale Kontraste in Anschlag zu bringen, zumal ein wesentlicher Teil

97 Vgl. Kriegel (2015, S. 25f.).

98 Vgl. Kriegel (2015, S. 32$)$. 
unseres Verständnisses darüber, was eine Intuition ist, darin besteht, sie mit anderen mentalen Zuständen zu kontrastieren. ${ }^{99}$ Kaum bestreitbar erleben wir es anders, eine Intuition in Bezug auf etwas zu haben als eine deduktiv erlangte Überzeugung mit demselben Gehalt zu haben. Was hier spezifisch für die Intuition ist, wurde mit den phänomenalen Eigenschaften Unmittelbarkeit und Gewissheit ausgemacht. Eine solide Verteidigung der Phänomenalität mentaler Zustände bedürfte weiterer Argumente, doch im Rahmen dieser Arbeit kann es dabei bleiben, auf die Plausibilität dieser Annahme für den mentalen Zustand der Intuition hinzuweisen.

Nehmen wir die Phänomenalität mentaler Zustände als gegeben an, lässt sich zweitens feststellen, dass die phänomenalen Merkmale dieser Zustände dem Erkenntnissubjekt dazu dienen können, sie von anderen mentalen $\mathrm{Zu}$ ständen zu unterscheiden (dies wurde mit der Überlegung des phänomenalen Kontrastes bereits angedeutet). Durch wiederholtes Erleben eines mentalen Zustandes kann ein Subjekt somit etwa feststellen: »Aha, jetzt habe ich wieder dieses Gefühl« oder »diese Art von Gedanke«, wobei das Subjekt mit `Gefühl oder >Art von Gedanke< im hier entwickelten Verständnis eine Intuition, also einen mentalen Zustand mit den phänomenalen Merkmalen der Unmittelbarkeit und Gewissheit bezeichnet. Doch hier stellt sich sofort eine kritische Frage: Ist ein solches Sich-Gewahr-Werden notwendig, um eine Intuition zu haben? Dies scheint in Bezug auf viele Verwendungsweisen von >Intuition<, gerade im psychologischen Bereich, nicht naheliegend. So würde man die Expertenintuition der Golfspieler, die »intuitiv« das passende Eisen wählen, wohl nicht erst als solche gelten lassen, wenn die Golfspieler sich dieser Intuition auf die erwähnte Weise bewusst würden. Doch betrachten wir diese Situation genauer: Was würde einen Golfspieler, der intuitiv Eisen 7 wählt, von einem Golfspieler, der unreflektiert (und dennoch nicht zufällig) Eisen 7 wählt unterscheiden, wenn sich der Golfspieler nicht aufgrund der phänomenalen Merkmale seiner Intuition bewusst wird? Ein solcher Unterschied liesse sich nicht angeben, was einem zu weiten und dadurch diffusen Intuitionsverständnis Tür und Tor öffnen würde. Das Sich-Gewahr-Werden und dadurch Individuieren der Intuition seitens des Subjekts soll daher Bestandteil des hier weiter auszuarbeitenden Intuitionsverständnisses sein. Diese Forderung steht im Einklang mit Erklärungsansätzen des Bewusstseins, welche das

99 David Pitt bringt die Bedeutung des phänomenalen Kontrasts auf den Punkt: It is only because type-distinct conscious thoughts have type-distinct phenomenologies (of the cognitive sort) that one can immediately distinguish them from each other. (Pitt 2004, S. 8) 
phänomenale Erleben als ihr Ausgangspunkt nehmen. ${ }^{100}$ In welcher Art sich das Sich-Gewahr-Werden genauer vollzieht, kann im Rahmen dieser Arbeit nicht weiter ergründet werden; hier sei auf die Literatur im Bereich der kognitiven Phänomenologie verwiesen. ${ }^{101} \mathrm{Zu}$ unterstreichen ist aber: Keineswegs wird eine Meta-Überzeugung gefordert (also die Überzeugung, dass ich jetzt eine Intuition habe). Auch davon abgesehen muss nicht zwingend ein von dem mentalen Zustand der Intuition isolierter kognitiver Vorgang angenommen werden; vielmehr kann das Sich-Gewahr-Werden bereits untrennbar mit dem mentalen Zustand verflochten sein, wie dies in der Tradition der Phänomenologie bereits seit Brentano und Husserl angenommen wird. ${ }^{102}$

Als Zwischenergebnis zur näheren Bestimmung der Intuition lässt sich also festhalten: Unmittelbarkeit und Gewissheit können weiterhin als zentrale Merkmale der Intuition gelten, indem sie als phänomenale Eigenschaften des mentalen Zustands der Intuition aufgefasst werden. Die Sichtweise der kognitiven Phänomenologie, wonach mentale Zustände phänomenale Eigenschaften haben, ist insbesondere für die Intuition plausibel, da ein wesentliches Element einer Intuition darin besteht, dass es sich auf eine bestimmte, wohl unverwechselbare Weise anfühlt, sie zu haben. Als wichtige Folge dieser Sichtweise lässt sich feststellen, dass ein Subjekt sich gewahr wird eine Intuition zu haben, wenn es eine Intuition hat. Dieses Sich-Gewahr-Werden wird in Bezug auf die Frage intuitive Rechtfertigung noch von Bedeutung sein.

\subsection{Intuition als Neigung zu einer Überzeugung}

Will man zu einem adäquaten Intuitionsverständnis kommen, also zu einem solchen, das der Phänomenalität der Intuition (Unmittelbarkeit, Gewissheit) Rechnung trägt, darf Intuition nicht ausschließlich als propositionale Einstellung, also als »Intuition, dass $\mathrm{p} \ll$, verstanden werden. Vielmehr ist für die Intuition zentral, was sich auf einer tieferen, nichtpropositionalen Ebene des Denkens abspielt. Um diese Annahme zu stützen und zugleich die $\mathrm{Zu}-$ sammenhänge zwischen der propositionalen und nichtpropositionalen Ebene der Intuition zu klären, sind zunächst Reduktionsversuche der Intuition auf Vermutungen oder Ahnungen zu prüfen und zurückzuweisen. Anschließend

\footnotetext{
100 Vgl. Kriegel (2009, u.a. S. 1-8); Smith (2005).

101 Neben der in diesem Kapitel bereits erwähnten Literatur sei auf die von Bayne und Montague (2011) sowie von Breyer und Gutland (2015) herausgegebenen Sammelbände hingewiesen.

102 Vgl. Zahavi (2006); Smith (2004, S. 76-121); Smith (2005).
} 
sollen Reduktionsversuche der Intuition auf Überzeugungen kritisch betrachtet und ebenso zurückgewiesen werden. Dadurch wird der Weg frei für die Ausarbeitung eines umfassenden Intuitionsverständnisses, welches sowohl die nichtpropositionale als auch die propositionale Ebene berücksichtigt.

Von Vermutungen (guess) oder Ahnungen (hunch) heben sich Intuitionen dadurch ab, dass sie keine bewusste Wahl darstellen. ${ }^{103}$ Ein Subjekt vermutet etwas aufgrund einer bestimmten Evidenz und leitet seine Vermutung in der Regel bewusst aus dieser Evidenz her. Eine Intuition jedoch stellt sich unweigerlich ein; das Subjekt erzeugt sie nicht bewusst. Daher wäre es seltsam zu sagen: »Ich vermute, dass p, aber ich weiß, dass nicht p «, während es durchaus möglich ist zu sagen: »Ich habe die Intuition, dass p, aber ich weiß, dass nicht $\mathrm{p} \ll$. Von Ahnungen unterscheiden sich Intuitionen durch die Gewissheit, die mit ihnen verbunden ist. George Bealer zeigt dies am Beispiel eines jungen Studenten, dem im Logik-Kurs folgende Frage gestellt wird: »Ist es eine logische Wahrheit, dass: wenn p oder q, dann ist es nicht der Fall, dass nicht $\mathrm{p}$ und nicht q?«. Der Student hat möglicherweise eine Ahnung, dass dies wahr ist, jedoch keine entsprechende Intuition. Hingegen erkennt dies jemand, der Erfahrung mit Logik hat, intuitiv. ${ }^{104}$ Durch diesen Unterschied hinsichtlich der Gewissheit sind Ahnungen »Intuitionen epistemisch vorgeordnet, insofern sie auf die nachträgliche Bestätigung oder Widerlegung durch den avisierten Sachverhalt angewiesen sind.«105

Zentrale doxastische Einstellungen, auf welche Intuition verschiedentlich zu reduzieren versucht wurde, sind Überzeugung und Urteil. ${ }^{106}$ Eine direkte und einfache Replik auf diese Reduktionsversuche lautet wie folgt: Es ist oft der Fall, dass man eine Intuition hat, dass p, ohne aber die Überzeugung zu haben, dass p. ${ }^{107}$ Als Beispiel hierfür können Fälle von kognitiver Verzerrung

\footnotetext{
103 Vgl. Bealer (1998, S. 210).

104 Vgl. Bealer (1998, S. 210).

105 Schildknecht (2003, S. 460) - Es sei jedoch daran erinnert, dass der Infallibilismus in Bezug auf Intuiton hier bereits zurückgewiesen wurde.

106 Auf die Unterscheidung zwischen Überzeugungen und Urteilen muss hier nicht näher eingegangen werden. Es soll gelten: Wenn ein Subjekt urteilt, dass p, dann ist es auch überzeugt, dass p. Im hier vorausgesetzten Wortsinn ist also die Überzeugung dem Urteil vorgelagert. Daher werden Positionen in Bezug auf Intuition als Überzeugung sowie als Urteil gemeinsam behandelt. Solche Positionen sind etwa die folgenden: Lewis (1983), der Intuitionen als Meinungen (opinions) verstanden wissen will; van Inwagen (1997, S. 309), der Intuitionen als Überzeugungen definiert; Gopnik und Schwitzgebel (1998, S. 77), die Intuitionen als eine Art von Urteilen verstehen; ebenso Ludwig (2007, S. 136f.) und Williamson (2007, S. 3).

107 Bealer (1998, S. 208) und Chudnoff (2013, S. 41-43) ziehen als Beispiel hierfür das Russellsche Paradoxon heran, mit dem die naive Mengenlehre als falsch erwiesen wird.
} 
wie etwa der sogenannte Spielerfehlschluss gelten: Dieser besteht darin, dass aus dem sehr häufigen Auftreten eines Ereignisses in der Vergangenheit darauf geschlossen wird, dass dieses Ereignis in Zukunft mit geringerer Wahrscheinlichkeit auftreten wird; also z.B.: »Der Würfel hat in den letzten 20 Würfen noch nie die 6 gezeigt, dann wird er sehr wahrscheinlich in den nächsten drei Würfen einmal die 6 zeigen.« Nun ist es möglich, dass eine über Statistik aufgeklärte Person zwar der richtigen Überzeugung ist, wonach die Wahrscheinlichkeit des künftigen Auftretens unbeeinflusst von den früheren Häufigkeiten ist, dies jedoch ihre Spielerfehlschluss-Intuition nicht zum Verschwinden bringt. Selbst einem professionellen Statistiker kann der Spielerfehlschluss intuitiv plausibel erscheinen. ${ }^{108}$ Ist diese Beschreibung korrekt, folgt daraus, dass Intuitionen nicht auf Überzeugungen oder Urteile reduziert werden können. ${ }^{109}$

Eine folgerichtige Präzisierung führt zu der Feststellung, dass Intuitionen nicht selber Überzeugungen (oder Proto-Überzeugungen) sind, jedoch Neigungen zu Überzeugungen konstituieren. ${ }^{110}$ Betrachten wir unter dieser Modifikation erneut den Spielerfehlschluss, so zeigt sich, dass das Problem der Intuition ohne Überzeugung nicht mehr auftaucht. Der Fall lässt sich nun wie folgt beschreiben: Das Subjekt hat durch die falsche Intuition die Neigung zu der Überzeugung, dass die Wahrscheinlichkeit der nächsten Würfe durch die Häufigkeit der vorangegangenen Würfe bestimmt wird. Jedoch kann diese Neigung von der gegenläufigen und richtigen Überzeugung übertrumpft werden, wonach ebendieser Zusammenhang nicht besteht. Ein Subjekt, das keine statistische Expertise hat, wird jedoch diese gegenläufige Überzeugung nicht haben. In diesem Fall ist es wahrscheinlich und folgerichtig, dass auf-

Jemand, der dieses Paradoxon versteht und zur richtigen Überzeugung gelangt, dass die naive Mengenlehre falsch ist, kann ohne weiteres noch die gegenläufige Intuition haben, dass die naive Mengenlehre korrekt ist. Die naive Mengenlehre behält, obgleich als falsch erkannt, ihre intuitive Plausibilität. Dieses Beispiel spricht ebenfalls gegen die weiter oben zurückgewiesene Gleichsetzung von Intuitionen mit Vermutungen.

108 Es ist empirisch bestätigt, dass solche Fälle falscher Intuitionen auch bei Experten auf dem entsprechenden Gebiet häufig vorkommen (vgl. Tversky/Kahneman 1974).

109 Die hier beschriebene Argumentation verläuft parallel zu einer entsprechenden Argumentation gegen den Doxastizismus im Bereich der visuellen Wahrnehmung: Mit Verweis auf Sinnestäuschungen wird gezeigt, dass eine Wahrnehmung bestehen bleibt, deren Gehalt dem Gehalt der zutreffenden Überzeugung widerspricht. Ein gutes Beispiel hierfür ist die Müller-Lyer-Täuschung, bei welcher die Länge zweier Linien zu vergleichen ist (vgl. Bealer 1998, S. 208; Chudnoff 2013, S. 27-31).

110 Im englischen Original, etwa bei Earlenbaugh/Molyneux (2009): inclination to believe. Damit verwandt und hier als gleichbedeutend betrachtet werden die Definitionen von Intuition als attraction to believe (Sosa 2007a, S. 62) oder disposition to judgment (Williamson 2007, S. 3). 
grund der Intuition - verstanden als Neigung zu einer Überzeugung - tatsächlich die falsche Spielerfehlschluss-Überzeugung gebildet wird. Diese Überzeugung behält die Charakteristik des Intuitiven, da sie die phänomenalen Eigenschaften der Unmittelbarkeit und Gewissheit aufweist: Die Überzeugung drängt sich dem Subjekt gewissermaßen auf, ohne dass es die Gründe hierfür benennen kann. Hier zeigt sich nun, dass die im vorigen Kapitel eingeführten phänomenalen Eigenschaften (Unmittelbarkeit, Gewissheit) präziser auf die intuitive Überzeugung zu beziehen sind, nicht auf die Intuition generell, welche in der hier erarbeiteten Terminologie die Neigung zu einer Überzeugung mit solchen phänomenalen Eigenschaften darstellt. Während bisher allgemein von der Intuition die Rede war, ist nun zu unterschieden zwischen der intuitiven Überzeugung und der ihr vorgelagerten nichtpropositionalen Intuition. Zur Nichtpropositionalität letzterer wird in Kürze mehr zu sagen sein.

Zuvor soll jedoch in Bezug auf die oben vorgenommene Modifikation sorgfältig betrachtet werden, was genau mit >Neigung zu einer Überzeugung`gemeint ist. Hier soll unter >Neigung` eine Disposition eines Subjekts verstanden werden, in bestimmten Situationen auf bestimmte Arten zu reagieren. Eine Neigung zu einer Überzeugung ist also einem Subjekt auf ähnliche Weise eigen wie eine Charaktereigenschaft (z.B. Großzügigkeit, Tapferkeit). ${ }^{111}$ Wer aufgrund seiner Intuition eine Neigung zu einer Überzeugung hat, wird demnach nicht zwingend die entsprechende Überzeugung ausbilden, wie auch eine großzügige Person nicht immer großzügig handelt; dies erfolgt erst durch die Überwindung zweier Hürden. Erstens sind Dispositionen konditional aufzufassen: Es wird immer eine Bedingung gedacht, die gegeben sein muss, damit die Disposition wirksam wird. Zweitens ist - selbst unter Erfüllung der genannten Bedingung - nicht jede Disposition derartig, dass sie sich mit Sicherheit aktualisiert. ${ }^{12}$ Die Neigung zu einer Überzeugung ist eine Disposition im Sinne der genannten zwei Hürden: Erstens erlangt das Subjekt die auf der Intuition beruhende Überzeugung nur, wenn überhaupt ein Prozess der Überzeugungsbildung in Gang kommt, ansonsten kann die Intuition auch auf einer nicht-doxastischen Ebene wirken, die noch näher zu bestimmen sein wird. Zweitens tritt bei gegebener Intuition eine der Intuition

\footnotetext{
111 Vgl. Sapire (2015).

112 Damit schließe ich mich Gilbert Ryles logischer Analyse des Dispositionsbegriffs an: Eine dispositionale Eigenschaft besitzen heißt nicht: in einem bestimmten Zustand sein oder eine bestimmte Veränderung durchmachen; es heißt vielmehr: sicherlich oder wahrscheinlich in einem bestimmten Zustand sein oder eine bestimmte Veränderung durchmachen, wenn eine bestimmte Bedingung erfüllt ist.

(Ryle 2015, S. 52)
} 
entsprechende Überzeugung zwar mit erhöhter Wahrscheinlichkeit, jedoch nicht mit Sicherheit auf. Einer der Faktoren, die dies verhindern können, ist das Vorliegen eines Anfechtungsgrunds (defeater)..13 Diese Bedingungen zeigen sich auch im Beispiel des Spielerfehlschlusses: Erstens kann eine Person dieser problematischen Intuition unterliegen, ohne jemals eine entsprechende Überzeugung gebildet zu haben; so kann sich die Intuition direkt in ihrem Spielverhalten zeigen. Zweitens kann eine Person zwar auf dem Weg der Überzeugungsbildung sein, jedoch die Überzeugung bewusst nicht bilden, weil sie sich einen Anfechtungsgrund vor Augen führt. Diese Beschreibung passt auf das Beispiel des Mathematikers, der zwar die intuitive Anziehung des Spielerfehlschlusses bemerkt, ihr aber aufgrund seines mathematischen Wissens nicht stattgibt.

Das hier vertretene dispositionale Verständnis von >Neigung zur Überzeugung < unterscheidet sich von anderen Konzeptionen, die im Zusammenhang mit der Intuition und der Wahrnehmung geltend gemacht wurden; so etwa von derjenigen Armstrongs:

What is an inclination to believe? I think it is nothing but a belief that is held in check by a stronger belief. We acquire certain beliefs about the world by means of our senses, but these beliefs are held in check by stronger beliefs that we already possess. So there is nothing here that is recalcitrant to an analysis of perception in terms of the acquiring of belief. ${ }^{114}$

Wenn wir eine Neigung zu einer Überzeugung wie Armstrong als Überzeugung verstehen, ergibt sich ein Problem: ${ }^{115}$ In vielen Fällen ist einerseits die falsche Wahrnehmung bereits recht deutlich, die korrekte gegenteilige Überzeugung sogar noch deutlicher. Wenn die beiden entgegengesetzten Neigungen nun als partielle Überzeugungen verstanden würden, dürften sie im Falle einer rationalen Agentin summiert einen Sicherheitsgrad von höchstens 1.o aufweisen. Wenn beispielsweise die Sicherheit in Bezug auf die partielle Überzeugung, dass die Müller-Lyer-Linien unterschiedlich lang sind, aufgrund des entsprechend starken Wahrnehmungseindrucks bei 0.7 liegt, dürfte die

\footnotetext{
113 Vgl. Schildknecht (2002, S. 161). Die weiter unten folgende Beschreibung der Intuition als maßgeblich nichtpropositionale Erkenntnisart wird diese dispositionale Analyse stützen.

114 Armstrong (1968, S. 221).

115 Vgl. Koksvik (2011, S. 52-55). Zu bemerken ist auch, dass diesem Verständnis zufolge Intuitionen rein propositional sind, da sie mit Neigungen zu Überzeugungen, die ihrerseits eine Art von Überzeugungen sind, identifiziert werden. Die Abgrenzung gegenüber Armstrongs >Neigung zur Überzeugung«-Konzeption hat also den zusätzlichen und großen Vorteil, dass Intuitionen nicht auf die propositionale Ebene begrenzt verstanden werden müssen. Warum dies ein Vorteil ist, wird sich im Folgenden zeigen.
} 
gegenteilige (z.B. aufgrund von Nachmessen zustande gekommene) Überzeugung, dass sie gleich lang sind, nur einen Sicherheitsgrad von 0.3 aufweisen. Wenn hingegen die Summe der Sicherheitsgrade mehr als 1 beträgt, ist die Rationalität des entsprechenden Subjekts in Frage zu stellen. ${ }^{116}$ Es ist aber nicht der Fall, dass Subjekte im beschriebenen Zustand rational kritisierbar sind. Es ist ihnen nicht als Fehler anzurechnen, wie sie die Müller-Lyer-Linien wahrnehmen. Somit ist die Ausgangsprämisse, nämlich die Definition der Wahrnehmung als Neigung zu einer Überzeugung in Armstrongs Sinn, zurückzuweisen. Ein analoges reductio-Argument lässt sich gegen den Versuch wenden, im Bereich der Intuition >Neigung zur Überzeugung< statt als vollständige als partielle oder Proto-Überzeugung zu verstehen. Der Kerngedanke ist, dass auch hier eine Agentin nicht rational kritisierbar dafür ist, dass sie wider besseres Wissen eine bestimmte Intuition hat (z.B. die SpielerfehlschlussIntuition), da sich diese Intuition - wie die Wahrnehmung einer optischen Täuschung - unweigerlich einstellt.

Diesem Problem lässt sich begegnen, indem die Intuition nicht als eine Art Proto-Überzeugung verstanden wird, die von anderen Überzeugungen in Schach gehalten wird. Vielmehr soll sie als eigenständiger mentaler Zustand gelten, der ein Subjekt geneigt macht, eine bestimmte Überzeugung zu haben. Es ist nicht der Fall, dass etwas, das einen geneigt macht eine Überzeugung zu haben, selber eine Überzeugung sein muss. Daher ist es präziser zu sagen, die Intuition konstituiert eine Neigung zu einer Überzeugung (statt: sie ist diese Neigung). Dies führt uns zu der oben vorgeschlagenen dispositionalen Analyse der Neigung zur Überzeugung zurück: Das Vorliegen der Intuition garantiert keine Überzeugung; erst unter weiteren Voraussetzungen wird das Auftreten der entsprechenden Überzeugung wahrscheinlich.

Einen weiteren Einwand gegen die Charakterisierung der Intuition als Neigung zu einer Überzeugung bringt George Bealer vor. ${ }^{117}$ Bealer macht geltend, dass er zahlreiche Neigungen zu Überzeugungen habe, beispielsweise in Bezug auf Zahlen, ohne die entsprechenden Intuitionen zu haben. Wer eine Intuition habe, erlebe dies als aktuelle kognitive Episode, während Neigungen zu Überzeugungen im Hintergrund bestehen können, wie auch die Überzeugungen selbst. Beispielsweise kann ich der Überzeugung sein, dass ich jünger als 60 bin, ohne in diesem Moment an meine Überzeugung zu denken. Dies ist Bealer zufolge bei der Intuition nicht der Fall. Man kann mir nur eine Intuition zuschreiben, wenn ich sie aktuell erlebe, somit seien Intuitionen nicht reduzierbar auf Neigungen zu Überzeugungen.

116 Vgl. Koksvik (2011, S. 55).

117 Vgl. Bealer (1998, S. 209). 
Auf diesen Einwand Bealers lässt sich erwidern: Keineswegs soll hier behauptet werden, dass alle Neigungen zu Überzeugungen auf Intuitionen zurückgehen. Sicherlich gibt es auch auf anderen Quellen beruhende Neigungen zu Überzeugungen, beispielsweise aufgrund von anderen Überzeugungen oder visuellen Wahrnehmungen. Es ist lediglich eine notwendige, keine hinreichende Bedingung dafür, eine Intuition zu haben, dass eine Neigung zur entsprechenden Überzeugung vorliegt. Bealer scheint zudem das Vorliegen von Neigungen zu Überzeugungen ohne Intuition als Indiz für den aktuell-auftretenden Charakter der Intuition (analog eines Urteils) im Gegensatz zum dispositionalen Charakter der Überzeugungen und Neigungen zu Überzeugungen zu nehmen. Wird das Vorliegen einer Neigung zu einer Überzeugung jedoch nur als notwendige Bedingung für eine Intuition anerkannt, lässt sich der Gegensatz zumindest auf dieser Grundlage nicht herleiten. Dies ist für das im Folgenden zu entwickelnde Intuitionsverständnis wichtig, da dieses mit einem eingegrenzten Verständnis der Intuition als aktuell-auftretende kognitive Episode nicht vereinbar ist.

In einem dritten Einwand gegen die Assoziation von Intuition und Neigung zu einer Überzeugung wird verneint, dass im Fall der Intuition notwendigerweise eine solche Neigung besteht. Diesen Einwand bringt Elijah Chudnoff in Analogie zu einer entsprechenden Überlegung im Bereich der visuellen Wahrnehmung vor. Dort sei, so Chudnoff, die folgende These mittlerweile weitgehend akzeptiert:

Possibly: $x$ has a perceptual experience representing that $p$ and $x$ does not judge, or have any inclination to judge, that $p .{ }^{118}$

Beispielsweise sei im Fall Müller-Lyer-Täuschung sehr wohl denkbar, dass jemand die beiden Linien als gleich lang wahrnehme, ohne auch nur die geringste Neigung zur Überzeugung zu haben, dass die beiden Linien gleich lang sind (da dieser Person bekannt ist, dass es sich um eine Täuschung handelt). ${ }^{119}$ Diese Debatte im Bereich der Wahrnehmung soll hier nicht

118 Chudnoff (2013, S. 28).

119 Als Reaktion auf diesen Einwand hat Kathrin Glüer (2009) vorgeschlagen, »Neigung zu einer Überzeugung « anders zu verstehen: Die Neigung beziehe sich nicht auf die Überzeugung, dass die Linien gleich lang sind, sondern auf die Überzeugung, dass die Linien gleich lang aussehen. Dieser Vorschlag führt aber nicht zum Ziel, da von der gegnerischen Seite mit treffenden Beispielen bestritten werden kann, dass selbst die von Glüer beschriebene Neigung immer besteht (vgl. Chudnoff 2013, S. 28f.). Die Überzeugungskraft von Glüers Vorschlag muss hier nicht abschließend beurteilt werden, da die Neigungs-These, auf direktere Weise - und begrenzt auf den Bereich der Intuition verteidigt wird. 
abschließend beurteilt werden. Vielmehr geht es um die Übertragung der Argumente auf den Bereich der Intuition, die kritisch betrachtet werden soll. Chudnoff behauptet, dass es sich hier ebenso verhalte: Es sei denkbar, dass jemand die Intuition hat, dass $\mathrm{p}$, ohne jedoch auch nur die geringste Neigung zur Überzeugung zu haben, dass p. ${ }^{120}$

Gehen wir zur Beurteilung dieser These auf den Spielerfehlschluss zurück: Laut der These wäre es denkbar, dass jemand diesem intuitiven Fehlschluss unterliegt, ohne auch nur die geringste Neigung zur Überzeugung zu haben, dass die vergangene Häufigkeitsverteilung die Wahrscheinlichkeit zukünftiger Ereignisse beeinflusst. Als Erwiderung auf Chudnoffs Einwand lassen sich zwei Punkte geltend machen: Erstens scheint Chudnoffs Einwand von einem Verständnis von >Neigung zu einer Überzeugung`auszugehen, welches von dem hier vertretenen dispositionalen Verständnis abweicht. Hat der Mathematiker wirklich keine Neigung zu einer der Spielerfehlschluss-Intuition entsprechenden Überzeugung? Dies ließe sich jedenfalls nicht daraus schließen, dass der Mathematiker ohne Zögern die entsprechende Überzeugung ablehnt. Vielmehr müssen gemäß dispositionaler Analyse zwei Bedingungen hinzukommen: Ein Prozess der Überzeugungsbildung muss überhaupt in Gang kommen und es dürfen keine Anfechtungsgründe bestehen. Beides ist im Mathematiker-Beispiel nicht anzunehmen. Zweitens ist zu berücksichtigen, dass ein Subjekt mehrere, einander widerstreitende Neigungen haben kann. Earlenbaugh und Molyneux bezeichnen diese als kompetitive Neigungen, die von Netto-Neigungen abzugrenzen sind (die Neigungen, die übrigbleiben, nachdem die kompetitiven Neigungen quasi den Kampf ausgefochten haben):

Opposing competitive inclinations can be co-occurrent in the agent - for example, one can have a competitive inclination to eat the cake and a simultaneous competitive inclination to stay on one's diet. Net inclinations, on the other hand, are what's left after the competitive inclinations have fought it out: The net inclination is the one that wins. ${ }^{121}$

Im Lichte des dispositionalen Verständnisses ist die Konzeption der >NettoNeigung zwar problematisch: Neigungen sollten immer als kompetitiv verstanden werden; was nach deren Konfrontation übrigbleibt, könnte z.B. ein Handlungsmotiv oder gegebenenfalls eine Überzeugung sein. Dennoch ist die Unterscheidung hilfreich um zu untermauern, dass kein zwingender Weg von den Neigungen zu entsprechenden Motivationen oder Überzeugungen führt.

\footnotetext{
120 Vgl. Chudnoff (2013, S. 41-44).

121 Earlenbaugh/Molyneux (2009, S. 106f.).
} 
Auf diese Differenzierung erfolgt eine Replik Chudnoffs, wonach sich ein Dilemma ergibt: Entweder sind die kompetitiven Neigungen bewusst oder unbewusst. Wenn sie unbewusst sind, dann sind sie nicht als Neigungen dieser Person zu betrachten. Wenn sie hingegen bewusst sind, dann sollte die Person wissen, dass sie diese Neigungen zur Überzeugung hat. ${ }^{122}$ Dies ist aber, wie postuliert wurde, nicht der Fall: Chudnoffs Seite insistiert, dass man Intuitionen haben kann, ohne sich einer Neigung zu einer entsprechenden Überzeugung bewusst zu sein.

Chudnoffs Replik überzeugt nicht; beide Hörner des Dilemmas sind angreifbar. So bleibt erstens unklar, warum eine unbewusste Neigung des Erkenntnissubjekts nicht als dessen Neigung zu bezeichnen sein soll. Betrachten wir Überzeugungen, die einer Person aktuell nicht bewusst sind: Jeder Mensch hat eine Vielzahl solcher Überzeugungen, an die er in einem gegebenen Moment nicht denkt. Niemand käme auf die Idee, diese Überzeugungen deswegen nicht als Überzeugungen der Person zu betrachten. Chudnoff würde hierauf möglicherweise den bereits oben erwähnten Punkt Bealers wiederholen, wonach es sich bei Intuitionen - im Gegensatz zu Überzeugungen um aktuell-auftretende kognitive Episoden handelt. Dies trifft jedoch die hier vorgebrachte Verteidigung nicht: Selbst wenn >Intuition< aktuell-auftretende Episode bezeichnet, kann sie Neigungen zu Überzeugungen konstituieren, die selber nicht solche Episoden sind. Es ist ein Kurzschluss, die Eigenschaften der kognitiven Episode (hier: der Intuition) mit den Eigenschaften der Disposition, die auf das Subjekt infolge dieser kognitiven Episode zutrifft, gleichzusetzen.

Auch das zweite Horn des Dilemmas lässt sich bestreiten. Sobald Chudnoff eine aktuell erlebte kognitive Episode als Intuition einordnet, hat er notwendigerweise eine entsprechende Neigung zu einer Überzeugung. Dies lässt sich durch kontrafaktische Szenarien plausibilisieren: Nehmen wir an, ich wüsste nichts über Statistik. Unter diesem Umstand scheint mir introspektiv auf der Hand zu liegen, dass ich, zumindest mit erhöhter Wahrscheinlichkeit, aufgrund der Spieler-Intuition die entsprechende falsche Überzeugung bilden würde. Dies hängt mit dem phänomenalen Merkmal der Gewissheit zusammen: Wenn dieses für die Intuition gilt, ist es schlicht unverständlich, was damit gemeint wäre eine Intuition zu haben, die keine Neigung zu einer Überzeugung (im dispositionalen Sinn) konstituiert.

Die These, wonach Intuitionen Neigungen zu Überzeugungen konstituieren, wurde derart ausführlich diskutiert und verteidigt, da sie den Boden für eine Analyse des nichtpropositionalen Erkenntnisfundaments der Intuition bereitet. Das dispositionale Verständnis dieser Neigungen erlaubt den Vorgriff

122 Vgl. Chudnoff (2013, S. 43). 
auf die Einsicht, wonach Intuitionen in einem engen Zusammenhang zu Überzeugungen stehen können, ohne damit Intuitionen auf Überzeugungen bzw. die Intuition allgemein auf eine propositionale Einstellung reduzieren zu müssen. So wird die philosophische Analyse an unser Erleben der Intuition anschlussfähig. Wird uns die Intuition bewusst, geschieht dies zwar oft im Modus der Überzeugung. In diesem Fall haben wir eine Überzeugung, ohne benennen zu können, woher die Gewissheit für diese kommt. Dies weist auf uns weitgehend verborgen bleibende kognitive Vorgänge hin, die offenbar die Gewissheit hervorbringen. (Hierin zeigt sich auch das zweite phänomenale Merkmal der Intuition: Unmittelbarkeit.) Woher kommt die Neigung zur Überzeugung und damit das Gewissheitserleben; wodurch wird dieses konstituiert? Diese Fragen sollen durch eine nähere Betrachtung der nichtpropositionalen Ebene der Intuition erhellt werden.

\subsection{Nichtpropositionale Ebene: Intuition als Typ 1-Prozess}

Die Auffassung, wonach der Gehalt einer Intuition stets propositional und dadurch begrifflich ist, wird im Folgenden als >Intuitions-Propositionalismus< bezeichnet. In der zeitgenössischen Intuitionsdebatte innerhalb der analytischen Philosophie sind alle maßgeblichen Positionen dem IntuitionsPropositionalismus verpflichtet. ${ }^{123}$ Dies äußert sich u.a. darin, dass in diesen stets von der >Intuition, dass p< die Rede ist, also von Intuition als einer propositionalen Einstellung, analog etwa zu einer Überzeugung. Dies würde implizieren, dass Intuitionen (als Gehalte der propositionalen Einstellung) ausschließlich begrifflich sind. Mit >begrifflich < ist hier gemeint, dass sich diese Gehalte vollständig in Begriffen spezifizieren lassen, über die das Subjekt verfügt. ${ }^{124}$ Aus Sicht der Intuitions-Propositionalisten sind Intuitionen

123 Beispielsweise vertreten Ernest Sosa und George Bealer vieldiskutierte und ansonst unterschiedliche Intuitionsverständnisse, die beide propositionalistisch sind: Sie sprechen von >Intuitionen, dass p<, von der Intuition als propositionale Einstellung und von Intuitionen als propositionale Gehalte (vgl. Sosa 2007b, S. 52; Bealer 1998, S. 207).

124 Bermúdez und Cahen nennen dies den conceptual constraint:

Specifications of the content of a sentence or propositional attitude should only employ concepts possessed by the utterer or thinker.

(Bermúdez/Cahen 2008, §2)

Von diesem in Fregescher Tradition stehenden conceptual constraint in Bezug auf propositionale Einstellungen soll hier ausgegangen werden. Präzisierend ist aber anzufügen: Wenn hier der Gehalt propositionaler Einstellungen als begrifflich bezeichnet wird, ist damit nicht gesagt, dass nichtpropositionale Einstellungen immer nichtbegrifflich sind. Es gibt nichtpropositionales begriffliches Wissen; nichtbegriffliche Erkenntnis fällt nicht 
also begriffliche Gehalte, die als solche nicht reichhaltiger als das begriffliche Repertoire des Subjekts sein können. ${ }^{125}$

Eine erste propositionalistische Reduktionsmöglichkeit wurde bereits zurückgewiesen: Intuitionen sind weder Überzeugungen noch ProtoÜberzeugungen, was sie unweigerlich in den Bereich des Propositionalen verschieben würde. Doch auch Verständnisse der Intuition als eigenständige Erkenntnisgattung sind oft propositionalistisch. Im Folgenden wird die Auffassung vertreten, dass der Propositionalismus in Bezug auf Intuition zu kurz greift und um eine nichtbegriffliche Ebene erweitert werden muss. Diese Erweiterung des Intuitionsverständnisses folgt Gottfried Gabriels allgemeinem Plädoyer zugunsten einer Erweiterung des Erkenntnisbegriffs von der propositionalen auf die nichtpropositionale Ebene. ${ }^{126}$ Die Begründung dafür erfolgt in zwei Schritten: Erstens kann mit Verweis auf den diesbezüglich analogen Fall der visuellen Wahrnehmung gezeigt werden, dass aus der Gängigkeit der Beschreibung >eine Intuition haben, dass $\mathrm{p}<$ nicht auf die generelle Propositionalität der Intuition geschlossen werden darf. Den dadurch entstandenen Raum gilt es in einem zweiten Schritt auszufüllen: Mit Verweis auf die kognitionswissenschaftliche Dual-Prozess-Theorie wird plausibel, dass sich Intuition maßgeblich im Bereich des Nichtpropositionalen abspielt und Intuitionen vorwiegend nichtbegrifflich sind.

In Bezug auf den ersten Schritt muss zwischen dem mentalen Zustand und dessen Gehalt differenziert werden. ${ }^{127}$ Diese Differenzierung wird im verbreiteten Sprachgebrauch oft nicht vorgenommen: Mit >Intuition< wird hier manchmal der mentale Zustand, manchmal dessen Gehalt bezeichnet. In letzterem Fall hat man aufgrund der sprachlichen Praxis oft propositionale Gehalte im Blick, wie auch im Fall der Wahrnehmungen und Überzeugungen ( $>$ S sieht, dass $\mathrm{p}<,>\mathrm{S}$ glaubt, dass $\mathrm{p}<$ ). Dadurch läuft man erstens Gefahr, die Propositionalität dieser Gehalte auf alle Gehalte des entsprechenden mentalen $\mathrm{Zu}$ stands zu übertragen, welche Generalisierung im Fall der Überzeugung, nicht aber in den Fällen der Wahrnehmung und der Intuition korrekt ist. Zweitens liegt der Fehler nahe, diese Generalisierung auch auf den mentalen Zustand selbst zu übertragen. Dagegen gilt auch für die Überzeugung als Paradefall

mit nichtpropositionaler Erkenntnis zusammen (vgl. Schildknecht 2003, S. 461; Gabriel 2015a, S. 62).

125 Vgl. Grajner (2011, S. 105f.).

126 Vgl. Gabriel (2015a).

127 William Lycan macht im Rahmen einer Untersuchung über moralische Intuitionen auf diesen Unterschied aufmerksam und führt die nützliche terminologische Differenzierung intuitings vs. intuiteds ein (vgl. Lycan 1988, S. 211; den Verweis darauf verdanke ich Earlenbaugh/Molyneux 2009, S. 97f.). 
einer propositionalen Einstellung: Überzeugungen haben propositionale Gehalte, sie sind jedoch nicht selber propositional. ${ }^{128}$ Dies zu sagen würde auf einen Kategorienfehler hinauslaufen, da >Begrifflichkeit $<$ - zumindest im hier verwendeten und philosophisch gebräuchlichen Verständnis - nur auf Gehalte sinnvoll anwendbar ist.

Doch auch wenn wir nur über die Gehalte der Intuition sprechen, besteht die Gefahr eines propositionalen Kurzschlusses. Dieser besteht darin die Aufmerksamkeit auf die propositionalen intuiteds zu richten und daraus zu schließen, dass alle intuiteds propositional sind. Ein solcher Intuitions-Propositionalismus lässt sich mit dem erneuten Verweis auf den Bereich der visuellen Wahrnehmung zurückweisen, in welchem eine Propositionalistin wie folgt argumentieren könnte: Der Vorgang der visuellen Wahrnehmung lässt sich stets ausdrücken mit `S sieht, dass $\mathrm{p}<$, also beispielsweise: $>S$ sieht, dass die Farbtafel grün ist . Somit sei klar, dass der Gehalt der visuellen Wahrnehmung nicht reichhaltiger sein könne als das begriffliche Repertoire des Subjekts. Nehmen wir zur Illustration dieses Punkts an, dass die Farbtafel den Farbton >grün 21< hat. Man könnte nun nicht sagen: >S sieht, dass die Farbtafel grün 21 ist $<$, wenn, wie hier angenommen werden soll, S nicht über den Begriff > grün 21< verfügt. Die Proposition wird durch das begriffliche Repertoire des Subjekts vollständig charakterisiert.

Diese Argumentation der Wahrnehmungs-Propositionalistin ist leicht angreifbar. Sie ist nicht überzeugend, weil wir `Sehen $<$ vom >Gesehenen< (d.h. vom propositionalen Gehalt) unterscheiden können. Damit ist klar, dass $>\mathrm{S}$ sieht, dass $\mathrm{p}<$ nur den begrifflichen Ausschnitt des Sehens wiedergibt, über den $\mathrm{S}$ in der Regel auch retrospektiv berichten kann (>Ich habe gesehen, dass die Farbtafel grün ist<). \Sehen dass $\mathrm{p}<$ ist nur ein Teil des Sehens, da wir immer mehr sehen als dasjenige, welches wir propositional festhalten können. ${ }^{229}$ Dieselbe Möglichkeit bleibt im Bereich der Intuition offen, da wir auch hier annehmen dürfen, dass $S$ immer mehr intuitiv erfasst als dasjenige, welches $S$ selber begrifflich festhalten kann. Beispielsweise ist es denkbar, dass S unter der intuitiven Annahme des Spielerfehlschlusses handelt, ohne dass sie dies selber so ausdrücken kann, da ihr die Begriffe der Häufigkeit und Wahrscheinlichkeit fehlen. Noch muss erwiesen werden, dass diese generelle Intuition vorwiegend mit nichtbegrifflichen Gehalten operiert; doch kann aufgrund der bisherigen

128 Vgl. Schildknecht (2003, S. 46o, Fn. 2). Schildknecht macht in diesem Zusammenhang darauf aufmerksam, dass die gängige Verwendung des Begriffs >propositionale Einstellung< irreführend ist.

129 Vgl. Heck (2000). 
Überlegungen bereits ausgeschlossen werden, dass Intuition a priori auf Propositionalität fixiert ist.

Um den nichtbegrifflichen Anteil der Intuition weiter auszuloten, lohnt es sich, auf psychologische und kognitionswissenschaftliche Befunde zurückzugreifen. Als erster Schritt hierzu kann eine Andeutung der weitum akzeptierten Dual-Prozess-Theorie dienen, innerhalb welcher die entsprechenden Befunde gut erklärt werden können. ${ }^{130}$ Die menschliche Kognition lässt sich gemäß Dual-Prozess-Theorien in zwei Typen der Informationsverarbeitung einteilen: ${ }^{131}$ Typ 1-Prozesse sind schnell, automatisch und haben eine hohe Kapazität der Informationsverarbeitung, während Typ 2-Prozesse langsam, intentional und hinsichtlich dieser Kapazität limitiert sind. ${ }^{132}$ Ein Vorzug der Typ 2-Prozesse ist, dass sie abstraktes Denken und explizites Schlussfolgern erlauben, was bei Typ 1-Prozessen nicht der Fall ist. Der Vorteil der Typ 1Prozesse ist ihre Schnelligkeit und hohe Verarbeitungskapazität.

Die unterschiedlichen Stärken der beiden Typen lassen sich anhand zweier Beispiele veranschaulichen: Auf der eine Seite steht der versierte Schachspieler mit seinen genau und weit im Voraus überlegten Zügen (Typ 2), auf der anderen Seite die Feuerwehrfrau, die in Sekundenschnelle realisiert, dass sie das brennende Gebäude verlassen muss (Typ 1). Die Feuerwehrfrau handelt gemäß verbreitetem Verständnis intuitiv. Wie ist dies beim Schachspieler? Sein Spiel als nicht-intuitiv zu bezeichnen wäre vorschnell, denn auch bei einem langsamen Spiel mit wohlüberlegten Zügen ist es durchaus denkbar, dass sich ein solcher Spieler bisweilen auf seine Intuition beruft, um Entscheidungen über die nächsten Züge zu treffen. Das eher langsame Tempo und der hohe Grad an diskursiver Reflexion im Schachspiel stellt nicht in Frage, dass sich auch ein Schachspieler an entscheidenden Stellen auf Typ 1-Denken stützt. Beispielsweise könnte ihm unmittelbar und gewiss erscheinen, dass er den Läufer auf Feld $\mathrm{C}_{7}$ verschieben sollte. Wie kommt diese Intuition zustande? Es ist wahrscheinlich, dass auch in diesem Fall die schnellen, unbewussten,

130 Es gibt viele unterschiedliche Dual-Prozess-Theorien; für eine Übersicht siehe Evans (2010b, S. 2of.). Die hier vorgenommene Darstellung beschränkt sich auf wenige zentrale Merkmale, um einen ersten Eindruck der kognitionswissenschaftlichen Basis zu vermitteln.

131 Die Bezeichnung $>$ Typ $<$ ist der in einigen Dual-Prozess-Theorien ebenfalls vorkommenden Bezeichnung `System $<$ vorzuziehen, da letztere suggeriert, dass es genau zwei kognitive Systeme der Informationsverarbeitung gibt, die sich mit dem diskursiven und intuitiven Denken decken. Dies ist nicht der Fall; vielmehr ist eine Vielzahl verschiedener kognitiver Systeme sowohl beim intuitiven als auch beim diskursiven Denken am Werk. Es ist somit sinnvoller, von zwei Typen kognitiver Prozesse zu sprechen (vgl. Evans 2010a, S. 315f.).

132 Vgl. Evans (2010b, S.20). 
mit hoher Verarbeitungskapazität operierenden Prozesse des Typ 1-Denkens am Werk sind.

Mit Blick auf solche Beispiele erstaunt es nicht, dass Typ 1-Denken in der psychologischen Literatur oftmals als >intuitives Denken< beschrieben wird. ${ }^{133}$ Dadurch scheint auf den ersten Blick eine Möglichkeit zu bestehen, die philosophische Studie für beendet zu erklären: Intuition wäre demnach nichts anderes als die Bezeichnung einer Art kognitiver Prozesse bzw. der Gehalte, die durch diese Prozesse hervorgebracht werden, nämlich ein Synonym für >Typ 1Denken $<$. Ein im Hinblick auf aktuelle Verwendungsweisen von >Intuition< adäquates Verständnis liesse sich dementsprechend rein psychologischkognitionswissenschaftlich ausarbeiten; philosophisch wäre kaum etwas beizusteuern.

Die beschriebene Sichtweise auf Intuition ist jedoch undifferenziert, da der Intuitionsbegriff ungebührlich geweitet wird, wenn alle Typ 1-Prozesse als $>$ Intuitionen< bzw. >intuitiv < bezeichnet werden. Typ 1-Denken ist beispielsweise auch bei automatisierten Prozessen wie Fahrradfahren oder Zähneputzen am Werk. Tatsächlich gibt es eine verbreitete Verwendungsweise, nach der solche Tätigkeiten als >intuitiv< bezeichnet werden können. Dies ist aber nicht die Intuition, die zu Beginn der vorliegenden Studie in den Blick genommen wurde. In dieser geht es vielmehr um Intuition im epistemischen Sinn. Daher muss ausgelotet werden, inwiefern Typ 1-Denken zu Erkenntnisprozessen beiträgt und wie sich dies auf die klassisch-philosophischen Fragen der Rechtfertigung und der Art der involvierten Gehalte auswirkt. Diese Eingrenzung wird zusätzlich von den Überlegungen zu den phänomenalen Merkmalen der Intuition unterstützt (siehe Kap. 3.1). Bei dem Typ 1-Denken handelt es sich um unbewusste oder zumindest schnelle und automatisierte Denkprozesse, die zu einem größeren Teil keine phänomenalen Eigenschaften aufweisen dürften, also mit keinem »wie ist es diesen Denkprozess zu durchlaufen« seitens des Subjekts. Mit einer psychologischen Beschreibung des Typ 1-Denkens kann es also nicht getan sein, wenn Intuition im hier interessierenden Sinn ergründet werden soll. Zweifellos spielt das Typ 1-Denken aber für die Intuition eine wichtige Rolle. Wie lässt sich diese scheinbare Spannung lösen? Dafür ist es höchste Zeit, den Intuitionsbegriff in Bezug auf die propositionale und nichtpropositionale Ebene sowie in Bezug auf die Akt-Gehalt-Unterscheidung zu entflechten.

Um den Zusammenhang zwischen Typ 1- und Typ 2-Prozessen und damit zwischen nichtpropositionalen und propositionalen Aspekten der Intuition zu präzisieren, ist zwischen verschiedenen Intuitionsbegriffen zu unterscheiden:

133 Vgl. Kahneman (2012); Gigerenzer (2007). 
- >Intuition< (im hier relevanten Sinn) bezeichnet eine Erkenntnisart, die vorwiegend auf Typ 1-Kognition beruht und epistemisch ausgerichtet ist.

- >intuitive Überzeugung bezeichnet eine Überzeugung, die sich aus der Intuition - also aus epistemisch ausgerichteter Typ 1-Kognition - nährt.

- >Intuition, dass p< bezeichnet eine propositionale Einstellung, die sich ebenfalls aus der Intuition nährt, deren Gehalt jedoch durch eine gegenläufige Überzeugung daran gehindert wird, zum Gehalt einer Überzeugung zu werden.

- >Nichtpropositionale Intuitionen< $\left(\right.$ Intuitionen $\left._{\mathrm{NP}}\right)$ bezeichnet die nichtpropositionalen und nichtbegrifflichen Gehalte, die durch die relevante Typ 1Kognition hervorgebracht werden. ${ }^{134,135}$

- >Propositionale Intuitionen< (Intuitionen ${ }_{\mathrm{P}}$ ) bezeichnet die begrifflichen Gehalte der >Intuitionen, dass p $<$, sowie der intuitiven Überzeugungen.

Diese Begriffe und damit verbundenen Unterscheidungen dürften anhand des Spielerfehlschluss-Beispiels besser verständlich werden: In jedem Fall besteht hier die Möglichkeit, dass die Intuition direkt Handlungen hervorruft, ohne dass dem Subjekt der Gehalt dieser Intuition begrifflich verfügbar wird - ein klarer Beleg dafür, dass Intuitionen nicht nur als propositionale Einstellungen verstanden werden dürfen. Beispielsweise ist denkbar, dass ein Subjekt aufgrund entsprechender Typ 1-Kognition im Roulette-Spiel nach jeder Runde die Zahl wechselt, auf die es seinen Spieleinsatz legt. In diesem

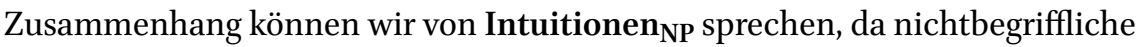
Gehalte, konstituiert durch Typ 1-Prozesse, die Grundlagen der Handlungen des Subjekts bilden. An diesem Beispiel zeigt sich nochmals deutlich, dass Intuitionen $_{\mathrm{NP}}$ keine Phänomenalität aufweisen, da das Sich-Gewahr-Sein in Bezug auf die Intuition hier fehlt.

Nun ist auch ein Subjekt denkbar, welches die Überzeugung hat, dass die bereits oft aufgetretenen Zahlen in den nächsten Runden mit geringerer Wahrscheinlichkeit auftreten werden. Diese nenne ich hier intuitive Überzeugung, da sie - wie wir annehmen wollen - auf der Intuition (im oben definierten Sinn) beruht. Dies zeigt sich dadurch, dass das Subjekt diese Überzeugung als unmittelbar und gewiss erlebt. Obwohl diese phänomenalen Eigenschaften

134 Propositionalität impliziert Begrifflichkeit; hingegen impliziert Nichtpropositionalität nicht Nichtbegrifflichkeit. Anders gesagt: Begrifflichkeit ist notwendige, aber nicht hinreichende Bedingung für Propositionalität (vgl. Schildknecht 2003, S. 463).

135 >Relevant< bedeutet hier, dass es sich um Typ 1-Denken handelt, welches sich auf den Bereich des Epistemischen bezieht; es geht um Gehalte, die Tatsachen in der Welt wiedergeben, und die - werden sie entsprechend verarbeitet - zu wahrheitsfähigen Überzeugungen werden können. 
erst Eigenschaften der intuitiven Überzeugung sind, verweisen sie zurück auf die Ebene des Typ 1-Denkens. Die Phänomenalität der intuitiven Überzeugung legt uns gewissermaßen eine Spur, die zu ihrer Herkunft aus der Typ 1-Kognition weist. So hat das Subjekt eine Überzeugung, ohne angeben zu können, woher sie kommt; sie ist beispielsweise nicht aus einer anderen Überzeugung abgeleitet.

Davon zu unterscheiden ist der Fall, in dem derselbe propositionale Gehalt involviert ist, jedoch das Subjekt zu diesem Gehalt nicht im Modus der Überzeugung steht. Diese Fälle möchte ich als solche bezeichnen, in denen das Subjekt eine Intuition, dass $\mathbf{p}$ hat. Wer beispielsweise Texte über den Spielerfehlschluss gelesen hat und dadurch weiß, dass dieser ein Trugschluss ist, kann trotzdem im Roulette-Beispiel die Intuition haben, dass die oft vorgekommene Zahl in der nächsten Runde mit geringerer Wahrscheinlichkeit vorkommen wird. Dies sind die Fälle, die wohl am Eindringlichsten zu einem Nachdenken über Intuition führen: Hier liegen die phänomenalen Merkmale der Unmittelbarkeit und Gewissheit vor, jedoch ohne dass wir die entsprechende Überzeugung haben. Sowohl im Fall der intuitiven Überzeugung als auch der >Intuition, dass $\mathrm{p}$ < sind die Gehalte propositional, es handelt sich also um Intuitionen ${ }^{1}{ }^{136}$

Nun, da die Differenzierung des Intuitionsbegriffs vorliegt, kann explizit gemacht werden, warum die Intuition bisher stets als mentaler Zustand bezeichnet wurde. Mit dieser Bezeichnung soll eine Abgrenzung gegenüber Positionen vorgenommen werden, welche die Intuition ausschließlich als episodisches und aus Sicht des Subjekts bewusst auftretendes Ereignis verstehen. ${ }^{137}$ Diese Auffassung ist nicht korrekt, da das schlicht als >Intuition< Bezeichnete sich bei näherer Betrachtung oftmals als eine Art der Überzeugung mit bestimmten phänomenalen Merkmalen, also eine intuitive Überzeugung entpuppt. Als Überzeugung muss sie somit dispositional sein. ${ }^{138}$ Mit Blick auf Beispiele ist dieses Verständnis durchaus plausibel: Wenn etwa die

136 Einzuräumen ist, dass diese Begrifflichkeit künstlich ist; so wird etwa das, was ich hier >intuitive Überzeugung« nenne, oft schlicht als >Intuition` bezeichnet. Mit der Wahl unterschiedlicher Begriffe soll verdeutlicht werden, dass es sich bei dem gemeinhin als >Intuition< bzw. >Intuitionen< Bezeichneten um verschiedene Phänomene handelt.

137 Vgl. u.a. Bealer (1998, S. 207) - siehe auch Kap. 3.2 in der vorliegenden Untersuchung.

138 Nach verbreiteter Auffassung sind Überzeugungen mentale Zustände, d.h., sie sind dispositional. Dies bringt es mit sich, dass Überzeugungen auch im Hintergrund bestehen können. Eine Überzeugung muss dem Subjekt zum jeweiligen Zeitpunkt nicht präsent sein, um ihm diese zuzuschreiben (vgl. Armstrong 1968, S. 213f.) . Ein Urteil dagegen ist als mentaler Akt dem Subjekt nicht nur gegenwärtig, es findet zum jeweiligen Zeitpunkt auch als Tätigkeit des Subjektes statt (vgl. Bealer 1998, S. 210). 
Feuerwehrkommandantin die intuitive Überzeugung hat, dass der Aufenthalt in den brennenden Gebäuden zu gefährlich ist, hat sie diese nicht nur im Moment, in dem ihr diese bewusst ist, sondern höchstwahrscheinlich auch zehn Sekunden später, wenn ihre Aufmerksamkeit nicht mehr auf die Überzeugung gerichtet ist. Dasselbe gilt für die >Intuition, dass $\mathrm{p}<$. Diese ist zwar keine Überzeugung, da sie angefochten wurde, bleibt aber ebenso dispositional: so etwa eine Spielerfehlschluss-Intuition, die einem Experten für Statistik bewusst werden kann. Auch hier liegt es auf der Hand, dass der Experte diese Intuition nicht nur in einem gegebenen Moment hat, in dem er über den Sachverhalt nachdenkt, sondern auch kurze (und längere) Zeit später, wenn er gedanklich mit etwas anderem beschäftigt ist. Alles andere scheint schlicht eine bizarre Beschreibung der Situation des Experten zu sein.

Betrachten wir nun die nichtpropositionale Ebene. Intuition als Art von Typ 1-Denkprozess, der Intuitionen ${ }_{\mathrm{NP}}$ hervorbringt, ist kein mentaler Akt, da Akte auf der personalen Ebene stattfinden müssen, um als solche bezeichnet zu werden. Die Typ 1-Denkprozesse sind selber keine Akte, wenn sie auch solche Akte eines Subjekts (z.B. Fahrradfahren) begleiten können. Allenfalls wäre dann die Typ 1-Intuition als mentales Ereignis zu beschreiben. Doch im seltensten Fall zielt die Beschreibung genau auf dieses Ereignis ab, sondern auch hier meist auf den Zustand, in welchem sich ein Subjekt aufgrund entsprechender Typ 1-Denkprozesse befindet. Beispielsweise ist die Person, die (im Unterschied zum Mathematiker im vorigen Beispiel) nicht an ihre Spielerfehlschluss-Intuition denkt, sondern direkt danach handelt, offenbar auf eine bestimmte Weise disponiert. Die Bezeichnung >mentaler Zustand passt also in jedem Fall am besten zur Intuition.

Das hier skizzierte Bild weist wesentliche Parallelen zu Positionen auf, welche die fundamentale Nichtpropositionalität der Wahrnehmung verteidigen. Wie bereits im Zusammenhang mit dem spropositionalen Fehlschluss angedeutet wurde, ist der Propositionalismus im Bereich der Intuition eine zum Propositionalismus im Bereich der Wahrnehmung analoge Verkürzung, da eine mögliche propositionale Einstellung zur allgemeinen These über die Propositionalität dieses Erkenntnisbereichs generalisiert wird. Demgegenüber lässt sich für beide Bereiche feststellen, dass im Fall der propositionalen Einstellung eine Bezugnahme auf einen Bereich erfolgt, der über die propositionale Struktur »ich habe die Intuition, dass p« bzw. »ich sehe, dass p « hinausgeht. Diese Feststellung wird - Argumenten im Bereich der Wahrnehmung entsprechend - u.a. aus den folgenden Gründen plausibel:

(1) Die Annahme einer fundamentalen Nichtpropositionalität der Intuition ermöglicht einen plausiblen Konjunktivismus: Wir können für wahre bzw. falsche propositionale Intuitionen (>intuitive Überzeugungen< bzw. 
>Intuitionen, dass $\mathrm{p}<$ ) eine gemeinsame Quelle angeben: die nichtpropositionale Intuition, d.h. eine Art des Typ 1-Denkens. ${ }^{139}$

(2) Die Annahme einer fundamentalen Nichtpropositionalität der Intuition ermöglicht die plausible, da einheitliche Beschreibung von Fällen, in denen Repräsentationen direkt zu Handlungen führen, ohne dass sich das Subjekt bewusst ist, dass es die jeweilige Intuition hat. Ein Beispiel hierfür ist der Spielerfehlschluss, der als Intuition behandelt werden kann, unabhängig davon, ob er dem Subjekt bewusst ist oder nicht.

(3) Die Annahme einer fundamentalen Nichtpropositionalität der Intuition ermöglicht eine plausible Erklärung des Geschehens im Fall einer zu Überzeugungen des Subjekts gegenläufigen Intuition (z.B. Spielerfehlschluss als Neigung zu einer Überzeugung, die durch eine andere Überzeugung ausgestochen wird): Wir können hier die intuitive Überzeugung, bzw. die >Intuition, dass $\mathrm{p}<$, als auf der nichtpropositionalen Intuition aufbauend begreifen.

\subsection{Intuitionen und mentale Modelle}

Die bisherigen Ausführungen haben eine Unterscheidung zwischen propositionalen und nichtpropositionalen Intuitionen (Intuitionen ${ }_{\mathrm{P}}$, Intuitionen $\mathrm{NP}_{\mathrm{NP}}$ ) nahegelegt. Intuition ist demnach eine primär nichtpropositionale Erkenntnisart, die auf der Ebene des Typ 1-Denkens anzusiedeln ist und deren Gehalte zunächst Intuitionen $_{\mathrm{NP}}$ sind. Allenfalls können durch Intuition Intuitionen ${ }_{P}$ hervorgebracht werden, wie durch Sehen propositionale Gehalte im Sinn von >Ich sehe, dass p< hervorgebracht werden können. Die Betrachtung dieser propositionalen Gehalte allein reicht aber nicht aus, um die Intuition als Erkenntnisart zu erfassen. Im nächsten Schritt soll daher auf die nichtpropositionale Ebene fokussiert werden: Was heißt es genau, wenn gesagt wird, Intuition bewege sich auf der Ebene des Typ 1-Denkens? Wenn damit, wie behauptet wurde, nicht einfach jede Art des Typ 1-Denkens gemeint sein soll, muss spezifiziert werden, was hier vonstattengeht. Dabei kommt eine plausible Erklärung nichtpropositionaler Intuition zustande, wenn auf die Theorie der mentalen Modelle zurückgegriffen wird. Diese Theorie ist dabei hilfreich, die phänomenale Ebene der Intuition (wie ist es, eine Intuition zu haben, Merkmale der Unmittelbarkeit und Gewissheit), die propositionale Ebene der Intuition (worauf berufen wir uns, wenn wir sagen: $>$ Ich habe die

139 Zum Konjunktivismus-Argument im Bereich der Wahrnehmung siehe Schildknecht (2002, S. 156f.). 
Intuition, dass p<) und kognitionspsychologische Befunde (v.a. im Rahmen der Dual-Prozess-Theorie) in Einklang miteinander zu bringen. Im Folgenden soll zunächst die Theorie der mentalen Modelle in ihren Grundzügen dargestellt werden. Anschließend kann gezeigt werden, inwiefern diese Theorie eine wichtige Grundlage zum Verständnis intuitiver Erkenntnis bildet.

Die Theorie der mentalen Modelle genießt in der aktuellen Psychologie hohe Akzeptanz. ${ }^{140}$ Wichtigster Vertreter und Wegbereiter der Theorie ist der Psychologe Philip Johnson-Laird. ${ }^{141}$ Johnson-Laird entwickelte die Theorie ab den 7oer-Jahren als Erklärungsansatz im Bereich des Schlussfolgerns, in Konkurrenz zu den bis dahin vorherrschenden Theorien der >mentalen Logik . Die Hypothesen, welche die Theorie der mentalen Modelle in Bezug auf menschliches Schlussfolgern aufstellt, bezogen sich in den ersten Arbeiten Johnson-Lairds auf explizites Schlussfolgern. Die mit der Theorie der mentalen Modelle verbundenen Hypothesen zeigten hierbei eine bessere Übereinstimmung mit empirischen Befunden als die Theorie der mentalen Logik. ${ }^{142}$ Eine zentrale solche Hypothese ist, dass sich das Schlussfolgern in zwei Schritten ereignet: In einem ersten Schritt konstruieren Subjekte mentale Modelle von Situationen, im zweiten Schritt entnehmen sie diesen Modellen die Folgerungen; sie lesen diese quasi von den mentalen Modellen ab (reading off ). ${ }^{143}$ Beispielsweise repräsentieren mentale Modelle, die von der visuellen Wahrnehmung ausgehen, welche Gegenstände im Blickfeld liegen (A liegt vor B und hinter C, etc.). Ebenso können mentale Modelle auf Basis von Beschreibungen und Wissen konstruiert werden, also intellektuell-abstrakt sein. Komplexere Aufgaben der Schlussfolgerung werden bewältigt, indem ausgehend vom Startpunkt (Wahrnehmungen, Erinnerungen, Annahmen, etc.,

\footnotetext{
140 Vgl. Rosa (2017, S. 117).

141 Siehe v.a. Johnson-Laid (1983).

142 Theorien der mentalen Logik gehen davon aus, dass sich Schlussfolgern anhand eines in der Kognition verankerten und implizit wirksamen logischen Kalküls vollzieht. Diese Theorien weisen unterschiedliche Schwachstellen auf, sowohl theoretischer als auch empirischer Art (d.h. in Bezug auf die Überprüfung ihrer Voraussagen). Hierzu zählt etwa die Erkenntnis, dass die logische Struktur vieler im Alltag vorkommender Folgerungen nicht nur von der syntaktischen Ebene abhängt. Zum Verständnis der Folgerung wird vielmehr zusätzliches Wissen benötigt. Um dieses zusätzliche Wissen nun mit der Folgerung zu verknüpfen, wird eine weitere Folgerung benötigt, was für die Theorie der mentalen Logik, die von einem »internen Kalkül« ausgeht, problematisch wäre, da hierfür wiederum syntaxbasierte Regeln feststehen müssten: Es droht ein infiniter Regress (vgl. Johnson-Laird 2010, S. 18243). Auf der empirischen Ebene weist die Theorie mentaler Logik ebenfalls Probleme auf. Dazu gehören Inhaltseffekte, die bei Experimenten wie dem Wason selection task auftraten (vgl. Johnson-Laird 1983, S. 29-34).

Vgl. Rosa (2017, S. 105).
} 
die ein mentales Modell konstituieren) weitere mentale Modelle entworfen werden, welche die Möglichkeiten, die konsistent mit dem entsprechenden Start-Modell sind repräsentieren. Aus den Modellen zieht das Subjekt Schlüsse. Für diese Art der Schlussfolgerung wird Typ 2-Denken gebraucht, da nur dieses aufgrund seiner Explizitheit mentale Modelle rekursiv ändern oder nach alternativen Modellen suchen kann. Die ursprünglichen mentalen Modelle und direkte »Ablesungen « von diesen Modellen - also unmittelbare Schlüsse, wie diese in Anlehnung an Descartes genannt werden können - sind jedoch im Bereich des Typ 1-Denkens zu verorten. ${ }^{144}$

Ein entscheidendes Merkmal der mentalen Modelle ist deren Ikonizität. Die Ikonizität ist es auch, welche die Theorie der mentalen Modelle anschlussfähig an das hier erarbeitete Intuitionsverständnis macht. Daher soll im Folgenden zunächst erläutert werden, was unter >Ikonizität < in Bezug auf mentale Modelle zu verstehen ist. Anschließend lässt sich die Verbindung dieses Merkmals zur intuitiven Erkenntnis aufzeigen.

Unter Ikonizität im hier gemeinten Sinn ist eine Eigenschaft von Zeichen, bzw. im Bereich des Mentalen von Repräsentationen zu verstehen, welche die Ähnlichkeit dieser Zeichen mit dem Bezeichneten (bzw. der Repräsentationen mit dem Repräsentierten) bedeutet. Diese Verwendung des Begriffs des Ikonischen geht auf die zeichentheoretischen Arbeiten Charles Sanders Peirces zurück, auf den sich Johnson-Laird explizit bezieht. ${ }^{145}$ Peirce grenzt ikonische von indexikalischen und symbolischen Zeichen ab. ${ }^{146}$ Beispielsweise ist eine kartografische Abbildung von Australien ein ikonisches Zeichen für Australien, da die kartografische Abbildung ungefähr die gleiche Form wie der Kontinent Australien aufweist. ${ }^{147}$ Indexikalische Zeichen hingegen haben eine direkte, in der Regel kausale Beziehung zum Bezeichneten, die im Moment der Repräsentation besteht. Das klassische Beispiel hierfür ist der Rauch als Zeichen für Feuer. Symbolische Zeichen schließlich haben eine rein konventionale Beziehung zum Repräsentierten. Beispielsweise ist das Wort $>$ Mensch< ein symbolisches Zeichen. ${ }^{148}$

144 Vgl. Johnson-Laird (2010, S. 18245).

145 Vgl. Johnson-Laird (2004, S. 181f.)

146 Die Definitionen bei Peirce:

An icon is a sign fit to be used as such because it possesses the quality signified.

An index is a sign fit to be used as such because it is in real reaction with the object denoted.

A symbol is a sign fit to be used as such because it determines the interpretant sign.

(Peirce, EP S. 307)

147 Vgl. Legg (2008, S. 209).

148 In vielen Fällen sind die Grenzen zwischen den Arten von Zeichen fließend; außerdem lassen sich Zeichen oft mehreren Arten zugleich zuordnen. Beispielsweise kann ein 
Johnson-Laird überträgt nun diesen zeichentheoretischen Begriff auf die Ebene des Denkens. Hierbei wird Ikonizität zu einem Merkmal der mentalen Modelle, die sich ihrerseits als strukturiertes Ensemble mentaler Gehalte (diese können z.B. Gehalte der visuellen Wahrnehmung sein, aber auch abstrakte Gehalte, die sich auf Sachwissen beziehen) verstehen lassen. Die Art, wie diese Gehalte in einem mentalen Modell strukturiert sind, macht dessen Ikonizität aus. Anders gesagt: Ikonizität ist ein Merkmal mentaler Modelle, welches sich auf die Struktur der in ihm angeordneten Gehalte bezieht. Die erwähnte Ähnlichkeitsrelation zeigt sich nun auch hier: nämlich zwischen der Struktur des mentalen Modells und der Struktur des Ausschnittes der Welt, auf den sich das mentale Modell bezieht. Im mentalen Modell findet sich dieser Weltausschnitt etwa so repräsentiert, wie auch ein Diagramm einen Sachverhalt repräsentiert: Den Relationen in der Welt entsprechen Relationen im mentalen Modell. Habe ich beispielsweise ein mentales Modell des Gebäudes, in dem ich mich zurzeit aufhalte, bedeutet dies, dass die tatsächlichen räumlichen Gegebenheiten (etwa, dass sich die Tür rechts von mir, ein Stockwerk über mir und eines unter mir befinden) in diesem Modell abgebildet werden, d.h. es gibt eine Struktur, welche die Relationen der Welt (rechts von mir, über mir,...) in ähnlicher Weise enthält.

Das im Kontext des Mentale-Modelle-Ansatzes wichtigste Merkmal des ikonischen Zeichens ist dessen Reichhaltigkeit. Durch diese Eigenschaft ermöglichen es ikonische Zeichen den Subjekten, auf ihre Repräsentationen zurückzukommen und neue Informationen über das Repräsentierte, die ihnen zunächst nicht zur Verfügung standen, im Repräsentat quasi abzulesen. Ein einfaches Beispiel für diesen Prozess der Neuerschließung in ikonischen Repräsentaten ist das Folgende: Jemand möchte prüfen, ob bestimmte Möbelstücke in seinem neuen Zimmer Platz finden. Er fertigt auf Basis des Möbelkatalogs und der Messungen im Zimmer einen Plan an (Grundriss und verschiebbare Möbel-Schablonen). Nun kann die Person diese Möbel auf dem Plan hin- und herschieben und dadurch eine Vielzahl neuer Informationen über die Situation der Möbel im Zimmer gewinnen. ${ }^{149}$

Piktogramm primär ikonisch sein, indem es etwa ein Flugzeug in stark schematisierter Form darstellt, jedoch auch als symbolisches Zeichen gelten, da es auf einer Konvention beruht (beispielsweise auf der Konvention, den Weg zum Flughafen auf diese Weise darzustellen). Ein Fußabdruck wiederum ist sowohl ikonisches als auch indexikalisches Zeichen, da er einerseits isomorph zum Fuß ist, andererseits in direkter kausaler Beziehung zu diesem steht (vgl. Legg 2008, S. 209).

149 Peirce weist mit einem ähnlichen Beispiel darauf hin, dass anhand von Diagrammen Operationen vorgenommen werden können, die neue Informationen über das Repräsentierte liefern: Ein General verwendet eine Landkarte während der Kriegsführung, 
Die für ikonische Zeichen charakteristische Reichhaltigkeit zeigt sich auch in den mentalen Modellen: Hier kann sie zu Schlussfolgerungen führen, die vorerst nur implizit in den mentalen Modellen enthalten waren. Dies wurde von Johnson-Laird und Mitarbeitenden in verschiedenen Experimenten nachgewiesen. In einer ersten Phase wurde dabei die Theorie der mentalen Modelle mit Studien untermauert, die das explizite Schlussfolgern der Versuchspersonen ausgehend von gegebenen Prämissen zu Konklusionen untersuchen. ${ }^{150}$ Die Grundidee ist, dass Individuen die Prämissen mit mentalen Modellen, also ikonisch, repräsentieren. Die mentalen Modelle erweisen sich dabei nicht nur für explizite Schlussfolgerungen als erklärungsmächtig, sondern auch für implizite. Johnson-Lairds Beispiel hierfür:

\begin{abstract}
Suppose, for example, you were to read in the paper: There was a fault in the signalling circuit. The crash led to the deaths of ten passengers ... then you might well infer that the passengers were killed in the crash. The text does not make this assertion [...]. Plainly, you jumped to a conclusion based partly on the content of the passage and partly on your general knowledge. You make such inferences automatically, almost involuntarily, and often without being aware of what you are doing. ${ }^{151}$
\end{abstract}

Auch hier wird die Situation, die im Zeitungsartikel beschrieben wird, durch ein mentales Modell repräsentiert, das die oben genannten Passagiere bereits als durch die Kollision getötet repräsentiert. Richtet das Subjekt seine Gedanken bewusst darauf, bemerkt es, dass es automatisch von diesem $\mathrm{Zu}$ sammenhang ausging und kann diesen Automatismus gegebenenfalls hinterfragen: Dadurch wird es sein mentales Modell mit Elementen ergänzen, die den Zusammenhang zwischen Kollision und Tod der Passagiere nicht aufweisen. Das Beispiel zeigt, wie Menschen mit ihrer begrenzten Kapazität der Informationsverarbeitung zu schnellen Schlussfolgerungen in der Lage sind. ${ }^{152}$ Die Theorie der mentalen Modelle kann somit sowohl unser explizites als auch unser implizites Schlussfolgern und sowohl unsere mögliche Korrektheit als auch unsere Fehleranfälligkeit in Bezug auf das Gefolgerte überzeugend erklären. ${ }^{153}$ Empirische Befunde stützen also die Theorie der mentalen Modelle.

um Positionen der eigenen Truppen und der Feinde darauf zu vermerken und die weiteren Strategien zu planen. Peirce spricht in diesem Zusammenhang von »experiments made upon diagrams « (Peirce, CP $§ 4.530$, S. 411f.).

150 Ein einfaches Beispiel: »Alle Künstler sind Bienenzüchter. Alle Bienenzüchter sind Chemiker. Folgt daraus: Alle Künstler sind Chemiker?«(Vgl. Johnson-Laird 1983, S. 94f.).

$15^{1}$ Johnson-Laird (1983, S. 127).

$15^{2}$ Vgl. Johnson-Laird (1983, S. 133).

153 Vgl. u.a. Johnson-Laird (1983), (2006), (2010). 
In einem nächsten Schritt soll der Zusammenhang von mentalen Modellen zu intuitiver Erkenntnis erarbeitet werden. Am besten gelingt dies anhand der Reflexion eines Beispiels, in dem die Erkenntnisleistung der Intuition klar zutage tritt:

\section{Feuerwehrkommandantin Claire:}

Claire arbeitet als Feuerwehrkommandantin. Aufgrund ihrer Arbeit hat sie viel Erfahrung mit Gefahrensituationen in Bezug auf Feuer. Nun wird sie mit ihrem Team zu einem anspruchsvollen Einsatz gerufen: Mehrere Gebäude eines komplex strukturierten Fabrikgeländes haben Feuer gefangen. Um das Feuer zu löschen, müsste Claires Team in das Innere der Gebäude gelangen. Claire hat nur wenig Zeit, um eine Entscheidung über das weitere Vorgehen zu treffen. Sie schaut sich kurz um und kommt unmittelbar zur Überzeugung, dass es noch zu gefährlich für ihre Leute ist, die Gebäude zu betreten.

Mit der Theorie der mentalen Modelle kann die Situation in Bezug auf das Schlussfolgern wie folgt erklärt werden: Claire hat ein mentales Modell (oder mehrere mentale Modelle in Kombination) der Situation an der Brandstätte. Dieses Modell wird sowohl durch Claires aktuelle Wahrnehmungen als auch durch ihre früheren Erfahrungen und ihr Hintergrundwissen gebildet. Entscheidend ist die Ikonizität des mentalen Modells: Die verschiedenen Wahrnehmungen, Erinnerungen und impliziten Wissenselemente treten bereits im Typ 1-Denken in einem ikonischen Zusammenhang auf, d.h. deren Struktur ist isomorph zur Struktur des Repräsentierten. Das heißt beispielsweise: Die Repräsentationen der Windrichtung und der Lokalität der Brandherde (beides vermittelt via visuelle und taktile Wahrnehmungen) werden in einem der Struktur der Realität entsprechenden Zusammenhang repräsentiert, so dass aus dieser ikonischen Repräsentation und allfälligen zusätzlichen Elementen dieses oder anderer mentalen Modelle das Typ 1-Denken die wahrscheinliche Weiterentwicklung des Brandes »ablesen« kann.

Das mentale Modell kann weder auf einzelne propositionale Gehalte noch auf singuläre Wahrnehmungen reduziert werden, da die strukturierte Anordnung der Elemente des Modells entscheidend ist: Beispielsweise beinhaltet Claires Modell die räumlichen Beziehungen der Gebäudeteile zueinander, wobei durch ihr gutes Raumvorstellungsvermögen auch nicht direkt sichtbare Gebäudeteile im mentalen Modell rekonstruiert werden. Ähnliche Strukturen finden sich im mentalen Modell bezüglich der Windrichtung, der Intensität des Feuers, etc. Claires Fähigkeit und Praxis, aus diesem mentalen Modell Erkenntnisse zu gewinnen, lässt sich mit Fug und Recht als Intuition bezeichnen. Das Beispiel plausibilisiert hierbei das oben dargestellte Intuitionsverständnis, welches Intuition nicht auf die propositionale Ebene reduziert, sondern 
verschiedene Möglichkeiten der Propositionalität bzw. Nichtpropositionalität offenlässt:

(1) Claire kann einen expliziten Gedanken fassen, indem sie zur Überzeugung kommt, dass es zu gefährlich für ihr Team ist, das Gebäude zu betreten (= intuitive Überzeugung).

(2) Als bereits propositionale Vorstufe dieser Überzeugung kann die Intuition $_{P}$ gelten: Claire hat die Intuition, dass es zu gefährlich für ihr Team ist das Gebäude zu betreten; ob sie dieser Intuition stattgibt und sie zur Überzeugung werden lässt (bzw. zu welchem Sicherheitsgrad) hängt davon ab, welche anderen Überzeugungen Claire hat, etwa bezüglich der Verlässlichkeit ihrer Intuition.

(3) In (1) und (2) war Typ 2-Denken involviert. Der ganze Vorgang kann aber auch auf der Ebene des Typ 1-Denkens bleiben, wenn Claires Repräsentation der Gefahr aus ihrem mentalen Modell entspringt und diese Repräsentation (Intuition ${ }_{N P}$ ) eventuell direkt handlungswirksam wird (z.B., wenn Claire ihre Leute zurückruft) ohne aber zur >Intuition, dass p p oder gar zu einer Überzeugung geworden zu sein. Diese Möglichkeit dürfte insbesondere bei Gelegenheiten, in denen sehr schnell reagiert werden muss, auftreten. Die Tatsache, dass auch hier relativ komplexe Situationen repräsentiert werden und Intuitionen ${ }_{N P}$ daraus entstehen können, wird mit der Theorie der mentalen Modelle, die eine Repräsentation dieser Komplexität und das »Ablesen « der Schlüsse daraus zulässt, überzeugend erklärt.

Die Theorie der mentalen Modelle lässt sich plausibel auf andere Beispiele der Intuition ausweiten und hilft bei der Erklärung verschiedener Aspekte, etwa in Bezug auf die Rechtfertigung und auf die Abgrenzung der Intuition zu anderen mentalen Zuständen. Dies wird sich im Lauf der weiteren Arbeit zeigen.

Als vorläufig letzter Punkt in Zusammenhang mit der Theorie der mentalen Modelle soll nochmals das Merkmal der Ikonizität hervorgehoben werden, da es für den Zusammenhang zur Intuition von entscheidender Bedeutung ist. Dies kann anhand des Beispiels der Feuerwehrkommandantin wie folgt dargestellt werden: Die in der aktuellen Situation entscheidenden Wahrnehmungen, Erinnerungen und impliziten Wissenselemente werden bereits im Typ 1-Denken in einen Zusammenhang zueinander gestellt, der ikonisch ist, d.h. der Struktur des Repräsentierten entspricht. Das heißt beispielsweise: die Windrichtung wird mit der Lokalität der Brandherde (vermittelt via schnelle Typ 1-Wahrnehmungen) in einen räumlichen Bezug gesetzt, so dass daraus die Weiterentwicklung des Brandes hervorgeht. Mit Blick auf solche Beispiele lässt sich die fundierende nichtbegriffliche Intuition genauer als Ableitung 
aus einem mentalen Modell beschreiben, das aufgrund seiner Ikonizität bestimmte Beziehungen (räumlich, zeitlich, logisch) der repräsentierten Wirklichkeit in sich enthält.

Die epistemologische Relevanz der mentalen Modelle wird im folgenden Teil der vorliegenden Arbeit genauer aufzuzeigen sein, in welchem die Gründe-Relation in Bezug auf Intuition näher betrachtet werden soll. Doch zuvor soll das bisher skizzierte Intuitionsverständnis gegenüber verbreiteten Alternativen abgegrenzt werden.

\subsection{Zurückweisung des Intuitions-Apriorismus}

Die Frage im folgenden Kapitel ist, ob Intuitionen ausschließlich Erkenntnisse a priori sind. Diese Auffassung wird in der zeitgenössischen analytischen Philosophie oftmals vertreten. Beispielsweise grenzt George Bealer die Intuition als rationale Intuition ein. Unter einer rationalen Intuition versteht Bealer dabei eine Intuition, die etwas als notwendig präsentiert, im Gegensatz zu einer >physikalischen Intuition<, der diese Notwendigkeit fehlt. Bealers Beispiel für eine physikalische Intuition ist die Intuition, dass ein untergrabenes Haus in sich zusammenfallen wird: Wohl nahezu alle Menschen haben diese Intuition, der entsprechende Sachverhalt ist aber nicht notwendig. Bealer sagt zwar nicht direkt, Intuition sei nur als rational zu verstehen, betont aber, dass ihn aposteriorische Intuitionen nicht interessieren. ${ }^{154}$

In der vorliegenden Arbeit soll Bealer und anderen Intuitions-Aprioristen in dieser Eingrenzung des Erkenntnisinteresses nicht gefolgt werden. Ein prima facie-Grund dafür ist, dass damit vieles, was im Alltagsgebrauch sowie in der psychologischen Forschung gemeinhin als Intuition verstanden wird, ausgeklammert werden müsste, ohne dass ein triftiger Grund für diese Ausklammerung vorliegt. Es ist lediglich das Interesse vieler Philosophen am Apriorismus, das eine Eingrenzung des Intuitionsverständnisses allenfalls rechtfertigt, nicht eine Besonderheit der Intuition selbst. Um diese These zu stützen, sollen im Folgenden zwei zentrale Motivationen für den Intuitions-Apriorismus kritisiert werden. ${ }^{155}$

154 Vgl. Bealer (1998, S. 207) - Weitere Autoren, welche die Intuition als rational definieren, sind z.B. Grundmann (2007), Pust (2000) und Sosa (1998; 2006b; 2007a; 2007b).

155 Die hier vorgebrachte Kritik richtet sich somit nicht nur gegen Positionen, die behaupten, dass Intuitionen immer a priori sind, sondern auch gegen solche, die zwar die Existenz von a posteriori-Intuitionen zulassen, a priori-Intuitionen aber für zentraler, höherrangig, eher zur wahren Erkenntnis führend, o.ä. erachten. 
Eine erste Motivation für den Intuitions-Apriorismus wurzelt in den zuvor in dieser Arbeit dargestellten Positionen, namentlich denjenigen Descartes' und Spinozas. Intuition wird hier als Königsweg zur Erkenntnis betrachtet und kann uns - so die Hoffnung dieser Philosophen - Einsicht in die Fundamente liefern, auf denen alle anderen Wahrheiten aufbauen. Intuition ist in dieser Betrachtung stets a priori. Im Gegensatz zu den unsicheren empirischen Inhalten ermöglicht sie uns den Zugang zu nicht-empirischen, unumstößlichen Wahrheiten, wie sie etwa in der Geometrie und Mathematik oder in Bezug auf selbstevidente Gesetze der Logik zu finden sind.

Dieses Intuitionsverständnis ist heute unhaltbar, wenn wir den psychologischen und umgangssprachlichen Intuitionsverständnissen Rechnung tragen wollen. Sobald wir Beispiele als möglich akzeptieren, in denen wir jemandem eine Intuition zuschreiben, die sich als falsch herausstellt, können wir das klassisch-philosophische Intuitionsverständnis nicht mehr in seiner vollen Tragweite akzeptieren. Als bestes Beispiel hierfür lässt sich der bereits erwähnte Spielerfehlschluss anführen: Selbst ein Experte für Statistik kann sich wohlmöglich der Intuition nicht erwehren, wonach vergangene Häufigkeiten künftige Wahrscheinlichkeiten beeinflussen, obwohl er sich deren Falschheit bewusst ist. ${ }^{156}$

Eine zweite Motivation für den Intuitions-Apriorismus hat ihre Wurzeln ebenfalls in der Philosophiegeschichte und ist mit der ersten verwandt. Hier geht es um die Sichtweise der Intuition als intellektuelle Wahrnehmung, wie sie sich bereits in der Wortherkunft intueri zeigt. Diese Tradition geht auf rationalistisch-idealistische Konzeptionen wie etwa diejenige Platons zurück. Die Intuition wird hier zum Pendant der sinnlichen Wahrnehmung im geistigen Bereich und soll fundamentale Erkenntnisse hervorbringen, die über den sinnlichen stehen. ${ }^{157}$

Wenn man Intuition als intellektuelles Pendant zur sinnlichen Wahrnehmung auffasst, ist dies mit der Grundcharakteristik der Intuition als unmittelbare Erkenntnis nicht vereinbar. Im Gegensatz zur Intuition beruft sich diskursive Erkenntnis auf Gründe, unter ihnen auch Wahrnehmungen.

${ }_{15}$ Ein analoges Beispiel stammt von George Bealer und bezieht sich auf die naive Mengenlehre (vgl. Bealer 1998, S. 208).

157 Dieser Zusammenhang lässt sich auch wie folgt ausdrücken: Für Rationalisten ergibt sich die Notwendigkeit, ihr Wissen mit Gehalten zu füllen. Während die Sinneswahrnehmung im Bereich des Empirischen die Gehalte liefert, wird die Intuition als >geistige Wahrnehmung<im Bereich des Intellektuellen herangezogen (vgl. Chudnoff 2013, S. 16). In diesem Denkschema bewegt sich auch Kant, wenn er, wie dargestellt wurde, die Intuition zunächst als geistige Wahrnehmung definiert, um deren Bestehen anschließend zurückzuweisen. 
Diese liefern - Kantisch gesprochen - den Inhalt für die empirischen Begriffe. Wenn einige Positionen nun die Intuition als ein Pendant dazu im Bereich des Rationalen auffassen, diskursivieren diese die Intuition und verlieren den Blick für deren tatsächliche Differenz zur diskursiven Erkenntnis. Der Einwand trifft solche Positionen im Sinne einer internen Kritik, da es bei der Unmittelbarkeit um eine breit anerkannte Grundcharakteristik der Intuition in Abgrenzung zur diskursiven Erkenntnis geht.

Die dritte Motivation für den Intuitions-Apriorismus ergibt sich aus einem methodischen Diskurs, in den sich die meisten Texte über Intuition innerhalb der analytischen Philosophie der letzten zwei Dekaden einordnen lassen. Es geht hierbei um die Infragestellung der traditionellen Autonomie der Philosophie durch naturalistische Ansätze, insbesondere durch die in jüngerer Zeit aufgekommene experimentelle Philosophie. ${ }^{158}$ Intuition wird in dieser Diskussion als zentrale Größe eines traditionellen Philosophieverständnisses eingeordnet. Dieses lässt sich etwas überspitzt wie folgt darstellen: Der traditionelle Philosoph sitzt im Lehnstuhl, sinniert von dort aus über Gedankenexperimente und baut auf den daraus entspringenden Intuitionen philosophische Argumente auf. Er hat kein Interesse an empirisch-wissenschaftlichen Befunden, weil diese keine Aufklärung über genuin philosophische Fragestellungen liefern. Diese können und müssen vielmehr vom Lehnstuhl aus, unter Berufung auf unsere Intuitionen, beantwortet werden. Das methodische Bekenntnis zur Intuition, die in diesem Verständnis als apriorisch und propositional aufgefasst wird, soll die Autonomie der Philosophie bekräftigen. ${ }^{159}$

Diese dritte Motivation für den Intuitions-Apriorismus lässt sich leicht aufgrund der Zielsetzung der vorliegenden Arbeit zurückweisen: Hier soll keine Argumentation für oder gegen die »Lehnstuhlthese« vorgebracht werden. (Die philosophiemethodische Intuitionsdiskussion wird aber im 6. Kapitel thematisiert.) Daher sollten wir aufgrund einer solchen Motivation keinen Intuitions-Apriorismus annehmen. Vielmehr ist ein Intuitionsverständnis, welches aposteriorische Intuition ebenfalls zulässt, die richtige Wahl, da allein dieses der Intuition als weitreichender und vielfältiger Erkenntnisart des Menschen gerecht werden kann. ${ }^{160}$

\footnotetext{
$15^{8}$ Vgl. u.a. Kornblith (1998), Weinberg/Nichols/Stich (2001), Weinberg et al. (2010), Knobe/ Nichols (2008).

159 Vgl. Bealer (1998, S. 201f.).

16o Die hier vorgebrachten Argumente gegen den Intuitions-Apriorismus wenden sich insbesondere auch gegen Positionen, die Notwendigkeit in ihre Intuitionsdefinition einbauen, wie etwa Ernest Sosa dies tut:

$S$ intuits that $\mathrm{p}$ if and only if S's attraction to assent to $<\mathrm{p}>$ is explained by four things in concert: (a) that $<\mathrm{p}>$ is simple enough, (b) that $\mathrm{S}$ understands it well enough,
} 


\subsection{Zurückweisung der Konzeption >Intuition als Erscheinung}

In der philosophischen Tradition wurde Intuition oftmals mit visueller Wahrnehmung verglichen. Parallelisierungen zur visuellen Wahrnehmung finden sich auch bei nahezu allen Autoren der Neuzeit, die im vorigen Teil der Arbeit besprochen wurden. Dies lässt sich durch die phänomenalen Eigenschaften der Intuition erklären: Ähnlich wie eine visuelle Wahrnehmung ist Intuition im Fall einer intuitiven Überzeugung - mit Unmittelbarkeit und Gewissheit verbunden. In beiden Fällen bleiben uns die Prozesse, die zur Wahrnehmung führen, verborgen. Gleichzeitig halten wir das Wahrgenommene (wie das Wort bereits andeutet) in der Regel für wahr, sofern eine Reflexion auf der propositionalen Ebene erfolgt. Dies ist auch bei optischen Täuschungen der Fall. Beispielsweise bemerken wir im Fall der Müller-Lyer-Täuschung aufgrund der persistierenden Erscheinung: Wir würden für wahr halten, dass die eine Linie länger ist als die andere, wenn uns die Täuschung nicht bekannt wäre. Allein diese phänomenalen Merkmale sind für die oftmaligen Vergleiche zwischen Intuition und Wahrnehmung verantwortlich; weitergehende Parallelisierungen sind hingegen zurückzuweisen. Dies soll im Folgenden durch eine kritische Untersuchung der Argumente George Bealers und Elijah Chudnoffs untermauert werden. ${ }^{161}$

Bealer versteht Intuitionen als intellektuelle Erscheinungen (intellectual seemings). Demzufolge gilt: Wenn jemand eine Intuition hat, dass $\mathrm{p}$, dann scheint es dieser Person, als ob p. Bealer meint also mit 〉Erscheinung< eine bewusste kognitive Episode, wie sie sich beispielsweise einstellt, nachdem man einen Augenblick lang eines der De Morganschen Gesetze der Logik betrachtet hat: Die Wahrheit des Gesetzes scheint evident. ${ }^{162}$ Mit der Charakterisierung der Intuition als Erscheinung grenzt Bealer diese zugleich von Überzeugungen ab. Dieses verdeutlicht er am Beispiel mathematischer Theoreme, von deren

(c) that $<\mathrm{p}>$ is either a modally strong proposition (necessarily true or necessarily false), or else a self-presenting proposition (attributing to $\mathrm{S}$ some current state of consciousness), and (d), that $<\mathrm{p}>$ is true.

(Sosa 2006b, S. 216)

Es ist nicht ersichtlich, warum sich eine als propositionale Einstellung verstandene Intuition nicht auf alle Arten von Gehalten, also auch auf nicht-notwendige, beziehen kann. Diese Kritik wird von Michael Lynch (2006, S. 230) an Sosa vorgebracht und passt zu den hier vorgebrachten Argumenten gegen den Apriorismus.

161 Vgl. Bealer (1998), Chudnoff (2013).

162 Vgl. Bealer (1998, S. 207). Die Übersetzung >Erscheinung< für seeming ist nicht sehr treffend und wird hier in Ermangelung eines passenderen Ausdrucks gewählt. Mit der obigen Beschreibung sollte ausreichend geklärt sein, was Bealer darunter versteht. 
Richtigkeit er durch deduktive Beweisführungen überzeugt wurde, für die er aber nach wie vor keine bestätigenden Intuitionen habe. ${ }^{163}$

Auch wenn Bealer darin zuzustimmen ist, dass Intuitionen nicht mit Überzeugungen gleichzusetzen sind, muss sein Verständnis der Intuition als intellektuelle Erscheinung zurückgewiesen werden. Ernest Sosa bringt einen überzeugenden Einwand gegen dieses Verständnis vor: ${ }^{164}$ Bei einer auf der Wahrnehmung basierenden Überzeugung haben wir zwischen der Tatsache in der Welt (z.B.: vor jemandem liegt ein roter, runder Apfel, den diese Person mit offenen Augen und bei gutem Licht sieht) und der Überzeugung (»Da liegt ein roter, runder Apfel«) etwas, das zwischen Tatsache und Überzeugung vermittelt: die Wahrnehmung von einem roten Apfel. Sosa zufolge fehlt uns diese Vermittlung im Bereich der Intuition. Wenn wir introspektiv betrachten, was bei einer Intuition vor sich geht, so stoßen wir auf nichts, was zwischen dem Fakt $1+1=2$ und der entsprechenden intuitiven Überzeugung vermitteln könnte. Im Fall der Intuition haben wir unmittelbar eine Überzeugung, ohne angeben zu können, wie wir auf diese gekommen sind und ob das mit der Überzeugung verbundene Gefühl der Gewissheit berechtigt ist.

Die Kritik Sosas kann wie folgt unterstützt werden: Einen Grund anzugeben würde im intellektuellen Bereich, in dem wir uns nun bewegen, bedeuten, eine begriffliche Schlussfolgerung herzustellen. Eine andere Möglichkeit ist nicht ersichtlich. Doch ist gerade das Fehlen einer solchen Schlussfolgerung das entscheidende Merkmal, welches das intuitive vom diskursiven Denken unterscheidet, wie bei Descartes' >ich denke, ich existiere < deutlich wurde. Hier gibt es zwei Wege, auf denen von >ich denke< auf >ich existiere < geschlossen werden kann: den diskursiven, bei dem ein deduktives Argument zwischen der ersten und der zweiten Aussage vermittelt; aber auch den intuitiven Weg aus den Meditationes, auf dem direkt aus >ich denke< >ich existiere< gefolgert wird. Bealer würde in diesem Beispiel wohl darauf beharren, dass der Grund für die Überzeugung >ich denke, ich existiere< eine dieser Überzeugung entsprechende intellektuelle Erscheinung ist. Doch ist diese intellektuelle Erscheinung etwas anderes als die unmittelbare und gewisse Überzeugung selbst? Die Verortung der Intuition als intellektuelle Erscheinung, also als Bindeglied zwischen Tatsache und Überzeugung, ist hier mit einem Dilemma konfrontiert: Entweder wird dieses Bindeglied im Sinne einer Deduktion gedacht, womit es jeglicher Evidenz, die überhaupt die Rede von Intuition motiviert hat, zuwiderläuft,

163 Vgl. Bealer (1998, S. 209).

164 Vgl. Sosa (2006b, S. 208). 
oder das Bindeglied löst sich in die - angeblich erst daraus folgende - Überzeugung auf. ${ }^{165}$

Vielleicht könnte Bealers Analyse der Intuition als intellektuelle Erscheinung der genannten Kritik noch standhalten, wenn sie nur auf Fälle bezogen würde, in denen ein Subjekt die Intuition hat, ohne die entsprechende Überzeugung zu haben; denn hier bliebe die Möglichkeit der obigen Kritik verwehrt, da keine Auflösung in eine Überzeugung stattfindet. Jedoch wäre eine solche Intuitionsanalyse äusserst unbefriedigend, da sie für den Fall der nicht in eine Überzeugung mündenden Intuition eine andere Erklärung als für den Standard-Fall heranziehen würde. Wenn Intuition im einen Fall nicht als intellektuelle Erscheinung gedeutet werden kann, sollte dies ohne zwingenden Grund auch nicht im anderen Fall getan werden.

Als weiterer Kritikpunkt an Bealers Konzeption lässt sich anfügen, dass schlicht unklar ist, was eine intellektuelle Erscheinung sein soll. Hier wird mit Analogien zur visuellen Wahrnehmung gearbeitet, die nie aufgelöst werden.

In einem neueren Ansatz vertritt Elijah Chudnoff die zu Bealer ähnliche These, wonach Intuition eine präsentative Phänomenologie (presentational phenomenology) aufweist. ${ }^{166}$ Dementsprechend seien Intuitionen sui generisErfahrungen:

Sui generist views of intuition are views according to which intuitions - like perceptual experiences - are pre-doxastic experiences that - unlike perceptual experiences - represent abstract matters as being a certain way. ${ }^{167}$

Im Gegensatz zur sui generis-Sicht steht Chudnoff zufolge die doxastische Sicht, wonach Intuitionen doxastische Einstellungen oder Dispositionen sind oder den Erwerb solcher Einstellungen bzw. Dispositionen bezeichnen. Im Rahmen seines suigeneris-Ansatzes verteidigt Chudnoff die Rolle der Intuition als Bindeglied zwischen Tatsache und Überzeugung. Sein zentrales Argument für diese Verteidigung geht von der Feststellung aus, dass es mehrere intuitive Wege gibt, um zur jeweiligen Überzeugung zu gelangen. Wenn die Intuition direkt die Überzeugung wäre, könnten wir dieser Tatsache nicht Rechnung tragen. Chudnoff verdeutlicht diesen Gedanken anhand eines Beispiels:

165 Eine ähnliche Kritik an der Auffassung von Intuition als intellektueller Erscheinung bringt Timothy Williamson in The Philosophy of Philosophy vor (vgl. Williamson 2007, S. 217).

166 Vgl. Chudnoff (2013, S. 9).

167 Chudnoff (2013, S. 25). 
Let $p$ be the proposition that $(a+b+c)^{3} \geq 27 a b c$ and $(a+b+c)^{3}=27 a b c$ just when $a=b=c$. Now consider two intuition experiences. In the first it intuitively seems to you that $p$ because you imaginatively manipulate a diagram that is a three-dimensional analogue of the two-dimensional diagram used earlier in the intuition that $(a+b)^{2} \geq 4 a b$ and $(a+b)^{2}=4 a b$ just when $a=b$. In the second it intuitively seems to you that $p$ because you reflect as follows: just as a big square of side $a+a$ exactly contains four $a \times a$ squares, so a big cube of side $a+a+a$ exactly contains twenty-seven $a \times a \times a$ cubes; the only thing that making the sides of those twenty-seven inner cubes unequal can do is leave spaces between them. Someone very good at visualizing might have the first experience. For the rest of us, the second is more likely. The two intuition experiences are phenomenally different: in each there is a distinctive way that $p$ intuitively seems true to you. This suggests that each includes more than an attitude (= intuitive seeming) and content $(=p) \cdot{ }^{168}$

Chudnoff zufolge wird im obigen Beispiel ersichtlich, dass wir Intuitionen als eigenständige, zwischen Tatsachen und Überzeugungen liegende Erscheinungen zu verstehen haben. Dies ist aus einem einfachen Grund nicht überzeugend: Die von Chudnoff geschilderten Visualisierungen sind nicht gleichzusetzen mit Intuitionen. Wenn ein Subjekt die geometrische Beweisführung durch visuelle Vorstellungen rekonstruiert, unterscheidet sich dies nicht in bedeutender Weise von einer direkten visuellen Rekonstruktion, die es beispielsweise vornimmt, wenn es den geometrischen Beweis skizziert. Warum sollte die Tatsache, dass jemand sich die Rekonstruktion bildlich vorstellt, statt sie mit Stift und Papier vorzunehmen, dazu führen, dass aus der Rekonstruktion eine Intuition wird? Dies scheint schlicht ad hoc und unplausibel zu sein.

Dieses Bedenken lässt sich noch weiterführen: Man stelle sich vor, jemand würde die Person, die zu der obigen mathematischen Einsicht kommt, fragen: »Wie bist du darauf gekommen?« Dann bestünde die Antwort dieser Person darin, zu erklären, was sie gesehen hat. Dass dies bloß vor dem inneren Auge geschah, ist nicht entscheidend. (Die Person könnte die geometrische Beweisführung nun auch auf einem Blatt skizzieren.) In jedem Fall würde die Person als Begründung für ihr Urteil auf ihre visuell erfolgte Beweisführung verweisen. Dies aber wäre ein diskursiver Begründungsvorgang. Die Tatsache, dass dieser geometrische Weg im Vergleich zu einer abstrakten mathematischen Beweisführung einfacher und visuell repräsentiert ist, vermag alleine noch nicht den geometrischen Begründungsweg als intuitiv zu erweisen. In Bezug auf das Beispiel bestünde eine intuitive Einsicht erst dann, wenn der Person die

168 Chudnoff (2013, S. 566 .). 
mathematische Aussage unmittelbar als gewiss erschiene; d.h., ohne dass sie einen Grund dafür angeben könnte.

Die Unterscheidung zwischen intuitivem und diskursivem Denken - so verschieden sie auch ausgestaltet wurde - hat sich durch alle im vorigen Teil dargestellten philosophischen Positionen gezogen und ist auch für das Alltagsverständnis von Intuition plausibel. Wer dieser Unterscheidung zustimmt, kann die Ablehnung des sui generis-Ansatzes nachvollziehen, da man sich hier auf ein Bindeglied zwischen Tatsache und Überzeugung berufen möchte, welches den Übergang rechtfertigt. Zur Rechtfertigung des Übergangs muss das Bindeglied als propositional aufgefasst werden; z.B. als >Erscheinen, dass $\mathrm{p}<$, welches zur >Überzeugung, dass p $<$ führt. Damit bewegen wir uns im Bereich eines diskursiven Begründungsvorganges und sind von einer Abgrenzung des Intuitiven gegenüber dem Diskursiven weit entfernt, womit auch die Motivation des sui generis-Ansatzes untergraben wird.

\subsection{Abgrenzung gegenüber Wahrnehmung}

An dieser Stelle scheint sich ein Spannungsfeld zwischen zwei bisher vertretenen Thesen zu ergeben: Einerseits wurde die Intuition $\mathrm{zu}$ mentalen Modellen in Bezug gesetzt, andererseits wurde der sui generis-Ansatz abgelehnt. Da Ikonizität ein wesentliches Merkmal mentaler Modelle ist, stellt sich die Frage, ob eine Theorie der Intuition, die auf mentale Modelle rekurriert, auch auf einen sui generis-Ansatz hinauslaufen könnte. Die Nähe von Ikonizität und Repräsentationalität der Wahrnehmung motiviert diese Frage. Eine genauere Untersuchung zeigt jedoch, dass eine auf mentalen Modellen basierende Theorie der Intuition vertreten werden kann, ohne in Richtung eines sui generis-Ansatzes gehen zu müssen. Entscheidend hierfür ist die Abgrenzung von mentalen Modellen zu Gehalten der Wahrnehmung, die hier kurz skizziert werden soll.

Ein wichtiges Unterscheidungsmerkmal zwischen mentalen Modellen und Wahrnehmung nennt Johnson-Laird:

Mental models should not be confused with visual images. A visual image represents how something looks from a particular point of view. It is an egocentric representation. In contrast, a mental model is an abstract structure [...]. It is not egocentric, but independent of point of view..$^{169}$

169 Vgl. Johnson-Laird (2006, S. 28). 
In diesem Sinn entspricht ein mentales Modell eines Zimmers nicht der visuellen Vorstellung, wie ich dieses Zimmer von einem bestimmten Standpunkt aus sehen würde, sondern eher einer ${ }_{3} \mathrm{D}$-Visualisierung des Zimmers durch ein Computerprogramm. ${ }^{170}$ Das Programm kann das Zimmer - gibt man einen bestimmten Betrachtungspunkt vor - in der entsprechenden Weise darstellen; in diesem Sinn bauen visuelle Vorstellungen oft auf mentalen Modellen auf, aber nicht umgekehrt. Hinsichtlich der Ikonizität entsprechen mentale Modelle visuellen Vorstellungen, hinsichtlich der Reichhaltigkeit jedoch nicht: Mentale Modelle - auch solche von visuellen, räumlichen Sachverhalten - enthalten mehr Information als diese, welche von einem bestimmten visuellen Betrachtungspunkt ausgehen. Dieser Erkenntnis entspricht auch das Beispiel der impliziten Raumorientierung: ein mentales Modell von dem mich umgebenden Raum zu haben heißt, diesen Raum - und somit auch mich - aus verschiedenen Perspektiven betrachten zu können. Diese Informationen sind implizit in meinem mentalen Modell enthalten und ermöglichen meine Orientierung im Raum.

Eine weiteres Abgrenzungsmerkmal mentaler Modelle gegenüber visuellen Vorstellungen besteht darin, dass mentale Modelle nicht rein ikonisch sind, sondern in vielen Fällen symbolische Anteile enthalten. Auch dieser Punkt knüpft an Peirce an, der den symbolischen Anteil bei Diagrammen erwähnt. Beispielsweise können wir den im folgenden Satz ausgedrückten Gehalt nicht durch eine visuelle (und somit auf ikonische Repräsentation beschränkte) Vorstellung repräsentieren: »Der Schrank befindet sich nicht hinter dem Klavier.« Das Problem einer rein visuellen Vorstellung ist hier die Negation. Diese muss symbolisch repräsentiert werden. ${ }^{171}$

Eine empirische Bestätigung für die Abgrenzung zwischen mentalen Modellen und visuellen Vorstellungen kann schließlich durch Experimente gewonnen werden, in denen die Leichtigkeit der Schlussfolgerungen - abhängig von visueller oder nicht-visueller Codierung - verglichen wird. In diesem $\mathrm{Zu}$ sammenhang ebenfalls zu erwähnen ist, dass bei visueller Vorstellung und mentalem Modellieren jeweils andere Hirnregionen aktiv sind. ${ }^{172}$

Diese Hinweise sollten genügen um zu zeigen, dass mentale Modelle nicht auf visuelle Vorstellungen reduziert werden können. Damit ist auch eine Hürde, welchementaleModelledaranhindernkönnte,nichtbloßDingewieBrandplätze (siehe das Beispiel der Feuerwehrkommandantin), sondern auch abstrakte

\footnotetext{
170 Vgl. Johnson-Laird (2006, S. 24-28).

171 Vgl. Johnson-Laird (2006, S. 32 ).

172 Vgl. Johnson-Laird (2006, S. 35f.).
} 
Sachverhalte (wie etwa die Französische Revolution) zu repräsentieren, überwunden. Zusätzliche Unterstützung erhält diese Überwindung, wenn man sich vor Augen führt, welche Vielzahl nicht-räumlicher struktureller Beziehungen zwischen Sachverhalten bestehen können; im Beispiel der Französischen Revolution etwa komplexe Ursache-Wirkungsbeziehungen oder normative Beziehungen zwischen verschiedenen Akteuren. Ein Modell kann ikonisch sein, also diese Beziehungen isomorph zu den entsprechenden Beziehungen der Wirklichkeit enthalten, ohne dadurch visuell sein zu müssen. Die Voraussetzung dafür ist, dass Symbole im Modell zugelassen sind (also Zeichen für >verursacht<, >ist grösser<, >ist nicht<, usw.).

Bei der Aufnahme von Symbolen in die mentalen Modelle zeichnet sich allerdings eine andere Schwierigkeit ab. Wie lässt sich die Nichtpropositionalität der mentalen Modelle, die im Einklang mit dem bereits entworfenen nichtpropositionalen Intuitionsverständnis steht, mit der Aufnahme von Symbolen vereinbaren? Es ist zumindest naheliegend, Symbole als begrifflich zu verstehen. Nun kommt hinzu, dass wahrscheinlich viele mentale Modelle, welche die Basis für Intuitionen bilden, solche Symbole enthalten: Kann etwa die Feuerwehrkommandantin das Feuer als nicht von Osten kommend repräsentieren, ohne über den Begriff `Osten $<$ und - noch vielmehr - über den Begriff >nicht $<$ zu verfügen? Dies sind berechtigte und schwierige Fragen, die an dieser Stelle nicht vollumfänglich beantwortet werden können. Es ist wohl sinnvoll, mentale Modelle als hierarchisch gegliedert zu verstehen. Auf der Mikroebene sind die Elemente der (auf Erfahrung beruhenden) mentalen Modelle maßgeblich nichtbegriffliche Erfahrungsgehalte. Auch basale strukturelle Beziehungen werden nichtbegrifflich repräsentiert, wie etwa im Beispiel der Raumorientierung. Kommen wir aber zu höherstufigen strukturellen Beziehungen, wie sie beispielsweise in Bezug auf die Repräsentation der Französischen Revolution maßgeblich sein könnten, scheint eine rein nichtbegriffliche Repräsentation nicht mehr wahrscheinlich.

Ein wichtiger Punkt muss jedoch in diesem Zusammenhang betont werden: Die basale Nichtpropositionalität der Intuitionen hängt nicht von einer durchgängigen Nichtbegrifflichkeit der mentalen Modelle ab. So ist es möglich, dass die Intuition der Feuerwehrkommandantin von gewissen Gehalten ausgeht, die ihr begrifflich zugänglich sein müssen: etwa die Repräsentation der Himmelsrichtung, aus der das Feuer kommt. Doch selbst wenn gewisse Ausgangspunkte im mentalen Modell begrifflich sind, können ausgehend von diesen Gehalten Ableitungen auf Typ 1-Ebene erfolgen, wie es bei der schnellen, nicht bewusst durchgeführten Ableitung im Beispiel der Feuerwehrkommandantin der Fall ist. Diese Ableitungen können zu nichtbegrifflichen Gehalten führen, also zu Intuitionen $_{\mathrm{NP}}$. 


\subsection{Abgrenzung gegenüber No Content-Ansätzen}

Viele Beispiele der Typ 1-Kognition ließen sich einfacher und dadurch scheinbar plausibler erklären, indem die relevanten repräsentationalen Vorgänge gar nicht als gehaltvoll angesehen würden. Solche No-Content-Ansätze werden etwa im Bereich der Tierkognition vertreten und scheinen dort oft überzeugend. ${ }^{173}$ Insofern scheint es naheliegend, diese Ansätze auch auf den Fall der Intuition zu übertragen. Lässt sich das auf mentalen Modellen basierende Intuitionsverständnis, bei dem repräsentationale Gehalte eine entscheidende Rolle spielen, vor dieser potentiellen Demontage retten?

In Bezug auf viele unserer Beispiele würde eine No-Content-Position insistieren, dass der Mentale-Modelle-Ansatz unplausibel ist, da in den entsprechenden Fällen viel wahrscheinlicher unmittelbare, selektionierte Reaktionen auf Umweltreize zu konstatieren seien als mentale Repräsentationen. Insbesondere Fälle, in denen Expertinnen aufgrund perzeptueller Informationen sehr schnelle Entscheidungen treffen (müssen), können plausibel so interpretiert werden, dass hier ein selektives Filtern von Umweltreizen stattfindet, welches durch Erfahrung oder gezieltes Training ermöglicht wurde. Solche Fälle können als Formen prozeduralen (vs. deklarativen) Wissens eingeordnet werden. ${ }^{174}$

Führt man diesen Gedankengang weiter, kommt man zur Position, wonach eine Vielzahl von Lebewesen immer und Menschen zumindest oft Situationen registrieren und darauf reagieren, ohne dass hierbei repräsentionale Gehalte im Spiel sind. Die Herausforderung für den hier vertretenen Mentale-ModelleAnsatz der Intuition besteht darin, durch das starke Betonen der Typ 1-Prozesse und der damit verbundenen Nichtbegrifflichkeit nicht auf einmal ohne Gehalt dazustehen. Wären No-Content-Ansätze auf Robotik oder Tierkognition beschränkt (in der sie schon längere Zeit erfolgreich operieren), würde die Abgrenzung zur Intuition vergleichsweise leichtfallen. Doch Vertreter des radikalen Enaktivismus und embodiment insistieren, dass auch im Bereich komplexer menschlicher Handlungen sowie der Wahrnehmung ohne die Idee der gehaltvollen Repräsentation vieles erklärt werden kann, was traditionellerweise als gehaltvoll galt. ${ }^{175}$

Als kritische Überlegung gegen No-Content-Ansätze lässt sich ins Feld führen, dass solche Ansätze im Gegensatz zum hier vertretenen MentaleModelle-Ansatz die Intuition nicht befriedigend einordnen können, da sie den

173 Vgl. Hutto/Myin (2013).

174 Vgl. Baber/Chen/Howes (2015).

175 Vgl. Hutto/Myin (2013, S. 42-50). 
erlebbaren Unterschied zwischen Intuition und basaleren Vorgängen nicht aufnehmen. Dies kann am Beispiel der kiki-bouba-Intuition gezeigt werden, welches aus einer Studie von Ramachandran und Hubbard stammt. ${ }^{176}$ Hugo Mercier und Dan Sperber verwenden dieses Beispiel, um für ihre These zu argumentieren, wonach verschiedenste inferentielle Module zu Outputs führen können, die wir mit dem gemeinsamen Begriff >Intuition<bezeichnen. ${ }^{177}$ Diese These scheint zwar überzeugend, der Intuitionsbegriff, den Mercier und Sperber zu deren Stützung verwenden, aber viel zu weit: Sie sprechen von Intuitionen als »conscious conclusions arrived at through unconscious processes «. ${ }^{178}$ Dies wird im Beispiel ersichtlich: Ramachandran und Hubbard präsentieren den Versuchspersonen ihres Experimentes zwei einfache und zugleich an nichts Bestimmtes erinnernde geometrische Figuren; die eine mit vielen spitzen Winkeln, die andere mit vielen Rundungen. Den Versuchspersonen wird gesagt, dass in einer fremden Sprache die eine Figur kiki, die andere bouba heiße und sie nun erraten sollen, welche Figur zu welchem Wort passt. 95 Prozent der Versuchspersonen ordnen kiki der spitzwinkligen, bouba der rundlichen Figur zu. Mercier und Sperber interpretieren dies so:

Ninety-five percent of people picked the left figure as kiki and the right as bouba. The strong intuition that it should be so is based, it seems, on a synesthetic association between sounds and shapes. Here intuition is close to perception. ${ }^{179}$

Diese Erklärung mit Rekurs auf den Intuitionsbegriff ist in der ursprünglichen Publikation zur Studie nicht zu finden. Vielmehr basiert Ramachandrans und Hubbards Hypothese auf einer evolutionär entwickelten Synästhesie:

Second, we propose the existence of a kind of sensory-to-motor synaesthesia, which may have played a pivotal role in the evolution of language. A familiar example of this is dance, where the rhythm of movements synaesthetically mimics the auditory rhythm. This type of synaesthesia may be based on cross-activation not between two sensory maps but between a sensory (i.e., auditory) and a motor map (i.e., Broca's area). This means that there would be a natural bias towards mapping certain sound contours onto certain vocalizations. ${ }^{180}$

$\mathrm{Zu}$ Recht taucht bei Ramachandran und Hubbard der Intuitionsbegriff nicht auf, wenn wir von einem phänomenal bestimmten Verständnis ausgehen, das

\footnotetext{
176 Ramachandran/Hubbard (2001).

177 Vgl. Mercier/Sperber (2017, S. 133ff.).

178 Mercier/Sperber (2017, S. 133).

179 Mercier/Sperber (2017, S. 134).

180 Ramachandran/Hubbard (2001, S. 19).
} 
Unmittelbarkeit und Gewissheit als zentrale Merkmale der intuitiven Überzeugung (bzw. der >Intuition, dass $\mathrm{p}<$ ) postuliert. Was in diesem Experiment geschieht, ist auf der kognitiven Ebene sicherlich erklärbar (eventuell via evolutionär entwickelter Synästhesie), jedoch nicht adäquat als Vorgang der Intuition beschreibbar. Dies wäre erst dann der Fall, wenn hier ein Gehalt abgeleitet würde (Intuition ${ }_{\mathrm{NP}}$ ), der eine Neigung zu einer intuitiven Überzeugung oder >Intuition, dass $\mathrm{p}<$ konstituiert - mit den entsprechenden phänomenalen Merkmalen. Würden wir spontane Assoziationen - etwa im synästhetischen Bereich - mit Intuitionen gleichsetzen, wäre einem ins Beliebige führenden Intuitionsbegriff Tür und Tor geöffnet, der jeden Typ 1-Vorgang als Intuition bezeichnet. Wahrscheinlich müssten dann - es sei denn, ein neues Abgrenzungskriterium würde gefunden - auch simple Reflexe und spontane Assoziationen jeglicher Art als Intuitionen bezeichnet werden. Man mag die phänomenale Charakterisierung der Intuitionen als unmittelbar und gewiss teilen oder nicht; in jedem Fall ist es wohl plausibel, dass zwischen Assoziationen in der Art von kiki-bouba und Beispielen, die in dieser Arbeit unter Intuition subsumiert werden, bedeutende Unterschiede bestehen, die nicht vernachlässigt werden sollten.

Sicherlich lässt sich nicht anhand eines einzelnen Beispiels unanfechtbar darauf schließen, dass alle No-Content-Ansätze den erwähnten Unterschied vernachlässigen. Zumindest jedoch haben No-Content-Ansätze die Tendenz, den Intuitionsbegriff zu unspezifisch werden zu lassen, da sie aufgrund des fehlenden Gehaltes keine Erklärung zur Hand haben, wie Intuitionen für Subjekte als solche erlebbar werden können. Zwar folgt aus dem Mentale-Modelle-Ansatz nicht, dass Intuitionen stets als solche erlebt werden; sie können ja auch auf der Typ 1-Ebene verbleiben. Doch wie gezeigt wurde, können Intuitionen durchaus bewusst und dadurch phänomenal charakterisierbar werden, indem sie als intuitive Überzeugungen bzw. >Intuitionen, dass $\mathrm{p}<$ auftreten. Wie kannn diese Erlebbarkeit - und damit die phänomenalen Merkmale der Intuition - erklärt werden, wenn keine Gehalte im Spiel sind? Der No-Content-Ansatz müsste hierauf eine gute Antwort geben; auch, um ein Ausufern des Intuitionsbegriffs zu verhindern. Solange diese Antwort nicht in Sicht ist, scheint ein überzeugender Ansatz zur Erklärung der Intuition nicht ohne repräsentationale Gehalte auszukommen.

Somit soll für die weiteren Untersuchungen am bisher erarbeiteten Intuitionsverständnis festgehalten werden, welches Intuition als primär auf der Ebene der Typ 1-Kognition operierende Erkenntnisart begreift, die Intuitionen ${ }_{N P}$ hervorbringt. Propositionale Verwendungsweisen von >Intuition< sind wichtig, müssen aber sorgfältig voneinander (intuitive Überzeugung, >Intuition, 
dass $\mathrm{p}<$ ) und von der nichtpropositionalen Ebene unterschieden werden. Aufgrund dieser geklärten Ausgangslage können nun die zentralen Fragen, die den Zusammenhang zwischen Intuition und epistemischer Rechtfertigung betreffen, angegangen werden. 